\title{
Synthesis of Generational Polyester Dendrimers Derived from Glycerol and Succinic or Adipic Acid
}

\author{
Michael A. Carnahan $†$ and Mark W. Grinstaff* \\ Departments of Chemistry and Biomedical Engineering, Boston University, Boston, MA \\ 02215. † Department of Ophthalmology, Duke University Medical Center \\ http://www.people.edu/mgrin
}

\section{Experimental}

All solvents were dried and freshly distilled prior to use $\left(\mathrm{CH}_{2} \mathrm{Cl}_{2}\right.$ with $\mathrm{CaH}_{2}$; THF with Na; pyridine with $\mathrm{CaH}_{2}$ ). All chemicals were purchased from Aldrich or Acros as highest purity grade and used without further purification (succinic acid 99\%; succinic anhydride 99\%; adipic acid 99\%; DCC 99\%, DMAP 99\%). All reactions were performed under nitrogen atmosphere. NMR spectra were recorded on a Varian INOVA 400, or a GE QE-300 (for HETCOR with APT) spectrometer. FT-IR spectra were recorded on a Nicolet Smart MIRacle Avatar 360 using a zinc selenide crystal. Chemical ionization mass spectra were obtained on a Hewlett Packard HP 5988A spectrometer using $\mathrm{NH}_{3}$. Fast atom bombardment mass spectra (FABMS) were obtained on a JOEL JMS-SX102A spectrometer using a 3-nitrobenzyl alcohol matrix. MALDI-TOF mass spectra were obtained using a PerSpective Biosystems Voyager-DE Biospectrometry Workstation operating in the positive ion mode using 2-(4-hydroxyphenylazo)-benzoic acid (HABA). Elemental analysis was obtained from Atlantic Microlab, Inc. Size exclusion chromatography was performed using THF as the eluent on a Polymer Laboratories PLgel $3 \mu \mathrm{m}$ MIXED-E column ( $3 \mu \mathrm{m}$ bead size) and a Rainin HPLC system (temp $=25$ ${ }^{\circ} \mathrm{C}$; flow rate $\left.=1.0 \mathrm{~mL} / \mathrm{min}\right)$. Polystyrene standards $(1 \mathrm{~K}, 4 \mathrm{~K}$, and $23 \mathrm{~K})$ were used for 
calibration. A TA Instruments Q1000 Modulated DSC was used to collect $\mathrm{T}_{\mathrm{m}}$ and $\mathrm{T}_{\mathrm{g}}$ data $\left(3{ }^{\circ} \mathrm{C} / \mathrm{min}, \pm 1{ }^{\circ} \mathrm{C} / 40 \mathrm{~s}\right)$. The $\mathrm{T}_{\mathrm{g}}$ values are reported as the average temperature of the thermal peak upon heating and cooling on the third cycle. Hydrodynamic radii were measured at $25{ }^{\circ} \mathrm{C}$ using a Wyatt Mini-Dawn Quasi-elastic Light Scattering (QELS) Instrument using a microCUVETTE measurement accessory. All solvents were filtered through 0.02 micron membrane syringe-tip filters.

Synthesis of 2-(cis-1,3-O-benzylidene glycerol)succinic acid mono ester (3) - cis-1,3$O$-Benzylideneglycerol $(9.90 \mathrm{~g}, 54.9 \mathrm{mmol})$ was dissolved in pyridine $(100 \mathrm{~mL})$ followed by the addition of succinic anhydride $(8.35 \mathrm{~g}, 83.4 \mathrm{mmol})$. The reaction mixture was stirred at room temperature for 18 hours before the pyridine was removed under vacuum at $40{ }^{\circ} \mathrm{C}$. The remaining solid was dissolved in $\mathrm{CH}_{2} \mathrm{Cl}_{2}(100 \mathrm{~mL})$ and washed three times with cold $0.2 \mathrm{~N} \mathrm{HCl}(100 \mathrm{~mL})$, or until the aqueous phase remained at $\mathrm{pH} 1$. The organic phase was evaporated and the solid was dissolved in deionized water $(300 \mathrm{~mL}) .1 \mathrm{~N}$ $\mathrm{NaOH}$ was added until $\mathrm{pH} 7$ was obtained and the product was dissolved in solution. The aqueous phase was extracted with $\mathrm{CH}_{2} \mathrm{Cl}_{2}(200 \mathrm{~mL})$ and then readjusted to $\mathrm{pH} 4$. The aqueous phase was subsequently extracted twice with $\mathrm{CH}_{2} \mathrm{Cl}_{2}(200 \mathrm{~mL})$, dried with $\mathrm{Na}_{2} \mathrm{SO}_{4}$, filtered, and evaporated. The solid was stirred in ethyl ether (50 mL) and cooled to $-25{ }^{\circ} \mathrm{C}$ for 3 hours before collecting $14.6 \mathrm{~g}$ of a white powder (95\% yield). ${ }^{1} \mathrm{H} \mathrm{NMR}$ $\left(\mathrm{CDCl}_{3}\right): \delta 2.68\left(\mathrm{~m}, 4,-\underline{\mathrm{H}}_{2}-\underline{\mathrm{CH}}_{2}-\right), 4.13\left(\mathrm{~m}, 2,-\underline{\mathrm{CH}}_{2}-\mathrm{CH}-\underline{\mathrm{C}}_{2}-\right), 4.33$ (m, 2, - $\underline{\mathrm{H}}_{2}-\mathrm{CH}-$ $\mathrm{C}_{2^{-}}$), 4.70 (m, 1, - $\left.\mathrm{CH}_{2}-\mathrm{CH}_{-}-\mathrm{CH}_{2}-\right), 5.51$ (s, 1, $\left.\mathrm{C} \underline{\mathrm{H}}\right), 7.34$ (m, 3, arom. $\left.\mathrm{C} \underline{\mathrm{H}}\right), 7.47$ (m, 2, arom. $\mathrm{C} \underline{\mathrm{H}}) .{ }^{13} \mathrm{C} \mathrm{NMR}\left(\mathrm{CDCl}_{3}\right): \delta 178.07(\underline{\mathrm{COOH}}), 172.38(\underline{\mathrm{COOR}}), 137.95(\underline{\mathrm{CH}}), 129.33$ $(\underline{\mathrm{CH}}), 128.51(\underline{\mathrm{CH}}), 126.26(\underline{\mathrm{CH}}), 101.43(\underline{\mathrm{CH}}), 69.15\left(\underline{\mathrm{CH}}_{2}\right), 66.57(\underline{\mathrm{CH}}), 29.24\left(\underline{\mathrm{CH}}_{2}\right)$, 
$29.05\left(\underline{\mathrm{CH}}_{2}\right)$. FTIR: $v\left(\mathrm{~cm}^{-1}\right) 2931$ (aliph. C-H stretch), $1713(\mathrm{C}=\mathrm{O})$. GC-MS $281 \mathrm{~m} / \mathrm{z}$ $\left(\mathrm{MH}^{+}\right)$(Theory: $280 \mathrm{~m} / \mathrm{z}\left(\mathrm{M}^{+}\right)$). Elemental Analysis C: $60.07 \%$; H $5.80 \%$ (Theory: C: $59.99 \%$; H $5.75 \%)$.

Synthesis of 2-(cis-1,3-O-benzylidene glycerol)adipic acid mono ester (6) - cis-1,3-Obenzylideneglycerol $(68.74 \mathrm{~g}, 0.54 \mathrm{~mol})$ was dissolved in pyridine $(150 \mathrm{~mL})$ followed by the addition of adipic anhydride $(82.5 \mathrm{~g}, 0.46 \mathrm{~mol})$. The reaction mixture was stirred at room temperature for 18 hours before the pyridine was removed under vacuum at $35^{\circ} \mathrm{C}$. The remaining solid was dissolved in DCM $(400 \mathrm{~mL})$ and washed two times with $0.2 \mathrm{~N}$ $\mathrm{HCl}(400 \mathrm{~mL})$, or until the aqueous phase remained at $\mathrm{pH} 1$. The organic phase was evaporated and the solid was added to deionized water $(300 \mathrm{~mL}) .1 \mathrm{~N} \mathrm{NaOH}$ was added until $\mathrm{pH} 7$ was obtained. The aqueous phase was washed with DCM $(400 \mathrm{~mL})$, to extract any remaining adipic anhydride, and then readjusted to $\mathrm{pH} 4$. The aqueous phase was subsequently extracted twice with DCM $(400 \mathrm{~mL})$, dried with $\mathrm{Na}_{2} \mathrm{SO}_{4}$, filtered, and evaporated to afford $67.53 \mathrm{~g}$ of a white powder (47.8 \% yield). ${ }^{1} \mathrm{H}$ NMR (400 MHz, $\left.\mathrm{CDCl}_{3}\right): \delta 1.70\left(\mathrm{~m}, 4,-\mathrm{CH}_{2}-\mathrm{C}_{2}-\mathrm{C}_{2}-\mathrm{CH}_{2}-\right), 2.35$ (m, 2, $\left.-\mathrm{C}_{2}-\mathrm{CH}_{2}-\mathrm{CH}_{2}-\mathrm{CH}_{2}-\right), 2.44$ (m, 2, - $\left.\underline{\mathrm{C}}_{2}-\mathrm{CH}_{2}-\mathrm{CH}_{2}-\underline{\mathrm{CH}}_{2}-\right), 4.13$ (m, 2, $\left.-\underline{\mathrm{C}}_{2}-\mathrm{CH}-\underline{\mathrm{C}}_{2}-\right), 4.25$ (m, 2, - $\left.\underline{\mathrm{C}}_{2}-\mathrm{CH}-\underline{\mathrm{C}}_{2}-\right), 4.67$

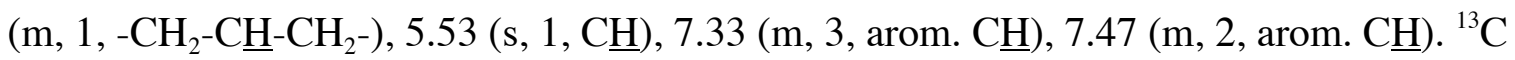
NMR (100.6 MHz, $\left.\mathrm{CDCl}_{3}\right): \delta 178.98(\underline{\mathrm{COOH}}), 173.48(\underline{\mathrm{COOR}}), 137.97(\underline{\mathrm{CH}}), 129.30$ $(\underline{\mathrm{C}}), 128.51(\underline{\mathrm{CH}}), 126.22(\underline{\mathrm{CH}}), 101.45(\underline{\mathrm{CH}}), 69.28\left(\underline{\mathrm{C}} \mathrm{H}_{2}\right), 66.13(\underline{\mathrm{CH}}), 34.13\left(\underline{\mathrm{CH}}_{2}\right)$, $33.71\left(\underline{\mathrm{CH}}_{2}\right), 24.43\left(\underline{\mathrm{CH}}_{2}\right), 24.21\left(\underline{\mathrm{CH}}_{2}\right)$. FTIR: $v\left(\mathrm{~cm}^{-1}\right) 3327(\mathrm{OH}), 2972$ and 2885 (aliph. C-H stretch), $1713(\mathrm{C}=\mathrm{O})$. FAB MS $309.1 \mathrm{~m} / \mathrm{z}[\mathrm{M}+\mathrm{H}]^{+}$(Theory: $308.3 \mathrm{~m} / \mathrm{z}[\mathrm{M}]^{+}$). Elemental Analysis C: $62.22 \%$; H $6.55 \%$ (Theory: C: $62.33 \%$; H $6.54 \%) . \mathrm{T}_{\mathrm{m}}\left({ }^{\circ} \mathrm{C}\right.$ ): 75.9, 79.5 at peak height. 
Synthesis of [G1]-PGLSA-bzld (9) - $\mathrm{Pd}(\mathrm{OH})_{2} / \mathrm{C}(10 \% \mathrm{w} / \mathrm{w})$ and activated carbon were added to a solution of [G0]-PGLSA-bzld $(3.57 \mathrm{~g}, 8.07 \mathrm{mmol})$ in THF $(25 \mathrm{~mL})$. The flask for catalytic hydrogenolysis was evacuated and filled with 60 psi of $\mathrm{H}_{2}$ before shaking for 10 hours. The catalyst and activated carbon were filtered off and washed with THF (50 mL). 2-(cis-1,3-O-benzylidene glycerol)succinic acid mono ester anhydride, 4, (21.99 g, $40.53 \mathrm{mmol})$ and then DMAP $(0.51 \mathrm{~g}, 4.21 \mathrm{mmol})$ were directly added to the deprotected core in the THF (more THF was added to give a total volume of $100 \mathrm{~mL}$ ). The reaction was stirred at room temperature for 14 hours under nitrogen atmosphere. Any remaining anhydride was quenched by the addition of n-propanol (4.0 $\mathrm{mL}, 44 \mathrm{mmol}$ ), which was allowed to stir for another 5 hours. The THF was removed under vacuum and the remaining contents were dissolved in DCM $(250 \mathrm{~mL})$ and washed once with $0.1 \mathrm{~N} \mathrm{HCl}(200 \mathrm{~mL})$ and three times with saturated sodium bicarbonate (200 $\mathrm{mL}$ ). The organic phase was dried with $\mathrm{Na}_{2} \mathrm{SO}_{4}$, filtered, and concentrated before the dendrimer was precipitated in hexanes $(450 \mathrm{~mL})$ and cooled to $-20{ }^{\circ} \mathrm{C}$ overnight. The hexanes were decanted and the precipitate was isolated to yield $10.29 \mathrm{~g}$ of a white solid (96.9\% yield). ${ }^{1} \mathrm{H} \mathrm{NMR}\left(\mathrm{CDCl}_{3}\right): \delta 2.58$ (m, 4, $\left.-\mathrm{C}_{2}-\mathrm{C}_{2}-\right), 2.63$ (m, 8, - $\left.\underline{\mathrm{CH}}_{2}-\underline{\mathrm{CH}}_{2}-\right), 2.71$ (m, 8, $\left.-\mathrm{C}_{2}{ }^{-} \underline{\mathrm{C}}_{2}-\right), 4.12\left(\mathrm{~m}, 12,-\underline{\mathrm{CH}}_{2}-\mathrm{CH}-\mathrm{C}_{2}-\right), 4.23$ (m, 12, $\left.-\underline{\mathrm{CH}}_{2}-\mathrm{CH}-\mathrm{C}_{2}{ }^{-}\right), 4.69$ (m, 4, - $\left.\mathrm{CH}_{2}-\mathrm{C} \underline{\mathrm{H}}-\mathrm{CH}_{2}-\right), 5.20$ (m, 2, - $\mathrm{CH}_{2}-\mathrm{C} \underline{\mathrm{H}}-\mathrm{CH}_{2}-$ ), 5.51 (m, 4, $\left.\mathrm{C} \underline{\mathrm{H}}\right), 7.33$ (m, 12, arom. $\mathrm{C} \underline{\mathrm{H}}$ ),

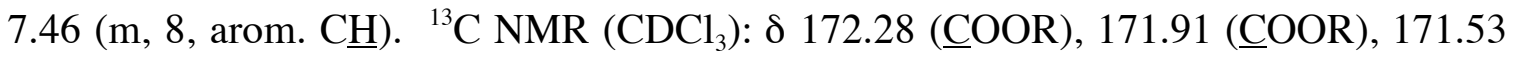
(ㅁOR), $138.03(\underline{\mathrm{C}}), 129.26(\underline{\mathrm{CH}}), 128.48(\underline{\mathrm{CH}}), 126.22(\underline{\mathrm{C}} \mathrm{H}), 101.32(\underline{\mathrm{CH}}), 69.50$ $\left.(\underline{\mathrm{C}} \mathrm{H}), 69.16\left(\underline{\mathrm{C}} \mathrm{H}_{2}\right), 66.54(\underline{\mathrm{CH}}), 62.49(\underline{\mathrm{CH}})_{2}\right), 29.36\left(\underline{\mathrm{CH}}_{2}\right), 29.03\left(\underline{\mathrm{CH}}_{2}\right)$. FTIR: v $\left(\mathrm{cm}^{-1}\right)$ 2858 (aliph. C-H stretch), 1731 (C=O). FAB MS $1315.6 \mathrm{~m} / \mathrm{z}\left(\mathrm{MH}^{+}\right)$(Theory: $1315.3 \mathrm{~m} / \mathrm{z}$ 
$\left(\mathrm{M}^{+}\right)$). Elemental Analysis C: $60.13 \%$; H $5.82 \%$ (Theory C: $60.27 \%$; H 5.67\%). SEC $\mathrm{M}_{\mathrm{w}}: 1460, \mathrm{M}_{\mathrm{n}}: 1450$, PDI: 1.01. $\mathrm{T}_{\mathrm{g}}\left({ }^{\circ} \mathrm{C}\right): 39.5$.

Synthesis of [G1]-PGLSA-OH (10) - Pd/C $(10 \% \mathrm{w} / \mathrm{w})$ was added to a solution of benzylidene protected [G1]-PGLSA $(0.27 \mathrm{~g}, 0.23 \mathrm{mmol})$ in THF $(20 \mathrm{~mL})$. The flask for catalytic hydrogenolysis was evacuated and filled with $50 \mathrm{psi}$ of $\mathrm{H}_{2}$ before shaking for 10 hours. The catalyst was filtered and washed with THF $(20 \mathrm{~mL})$. The filtrate was evaporated to give $0.178 \mathrm{~g}$ of a colorless, viscous oil (94\% yield). ${ }^{1} \mathrm{H} \mathrm{NMR}\left(\mathrm{CD}_{3} \mathrm{OD}\right): \delta$ $2.63\left(\mathrm{~m}, 20,-\mathrm{C}_{2}-\mathrm{C}_{2}-\right), 3.52\left(\mathrm{~m}, 4,-\underline{\mathrm{C}}_{2}-\mathrm{CH}-\mathrm{C}_{2}-\right), 3.64$ (m, 8, - $\left.\underline{\mathrm{C}}_{2}-\mathrm{CH}-\underline{\mathrm{C}}_{2}-\right), 3.80$ (m, 2, - $\left.\mathrm{CH}_{2}-\mathrm{C} \underline{\mathrm{H}}-\mathrm{CH}_{2}-\right), 4.05$ (m, 2, - $\left.\underline{\mathrm{CH}}_{2}-\mathrm{CH}-\underline{\mathrm{C}}_{2}-\right), 4.14$ (m, 2, - $\left.\underline{\mathrm{C}}_{2}-\mathrm{CH}-\mathrm{C}_{2}-\right), 4.21$ (m, 4, - $\left.\underline{\mathrm{CH}}_{2}-\mathrm{CH}-\mathrm{C}_{2}{ }^{-}\right), 4.30\left(\mathrm{~m}, 4,-\underline{\mathrm{CH}}_{2}-\mathrm{CH}-\mathrm{C}_{2}{ }^{-}\right), 4.85\left(\mathrm{~m}, 2,-\mathrm{CH}_{2}-\mathrm{C} \underline{\mathrm{H}}-\mathrm{CH}_{2}-\right), 5.25$ (m, 2, -

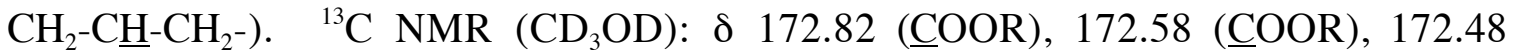
(ㅁOR), 172.08 (ㅁO), $75.82(\underline{\mathrm{CH}}), 69.90(\underline{\mathrm{CH}}), 69.68(\underline{\mathrm{C}} \mathrm{H}), 65.66\left(\underline{\mathrm{CH}_{2}}\right), 62.85$ $\left(\underline{\mathrm{C}} \mathrm{H}_{2}\right), 62.30\left(\underline{\mathrm{CH}}_{2}\right), 60.43\left(\underline{\mathrm{CH}}_{2}\right), 28.83\left(\underline{\mathrm{CH}}_{2}\right), 28.61\left(\underline{\mathrm{CH}}_{2}\right)$. FTIR: v $\left(\mathrm{cm}^{-1}\right) 3405(\mathrm{OH})$, 2943 (aliph. C-H stretch), 1726 (C=O). FAB MS $963.2 \mathrm{~m} / \mathrm{z}\left(\mathrm{MH}^{+}\right)$(Theory: $962.9 \mathrm{~m} / \mathrm{z}$ $\left(\mathrm{M}^{+}\right)$). Elemental Analysis C: $47.13 \%$; H $6.11 \%$ (Theory C: $47.40 \%$; H $6.07 \%$ ). SEC $\mathrm{M}_{\mathrm{w}}: 1510, \mathrm{M}_{\mathrm{n}}: 1500$, PDI: 1.01. $\mathrm{T}_{\mathrm{g}}\left({ }^{\circ} \mathrm{C}\right):-20.2$.

Synthesis of [G2]-PGLSA-bzld (11) - $\mathrm{Pd}(\mathrm{OH})_{2} / \mathrm{C}(20 \%$ w/w) and activated carbon were added to a solution of [G1]-PGLSA-bzld $(4.40 \mathrm{~g}, 3.43 \mathrm{mmol})$ in THF $(50 \mathrm{~mL})$. The flask for catalytic hydrogenolysis was evacuated and filled with 60 psi of $\mathrm{H}_{2}$ before shaking for 10 hours. The catalyst and activated carbon were filtered off and washed with THF (50 mL). 2-(cis-1,3-O-benzylidene glycerol)succinic acid mono ester anhydride $(18.46 \mathrm{~g}, 34.02 \mathrm{mmol})$ and then DMAP $(0.831 \mathrm{~g}, 6.80 \mathrm{mmol})$ were directly added to the deprotected dendrimer in the THF. The reaction was stirred at room 
temperature for 14 hours under nitrogen atmosphere. Any remaining anhydride was quenched by the addition of n-propanol $(3.0 \mathrm{~mL}, 33 \mathrm{mmol})$, which was allowed to stir for another 5 hours. The THF was removed under vacuum and the remaining contents were dissolved in DCM $(400 \mathrm{~mL})$ and washed once with $0.1 \mathrm{~N} \mathrm{HCl}(300 \mathrm{~mL})$ and three times with saturated sodium bicarbonate $(300 \mathrm{~mL})$. The organic phase was dried with $\mathrm{Na}_{2} \mathrm{SO}_{4}$, filtered, and concentrated before the dendrimer was precipitated in hexanes $(900 \mathrm{~mL})$ and cooled to $-20{ }^{\circ} \mathrm{C}$ overnight. The hexanes were decanted and the precipitate was isolated to yield $9.85 \mathrm{~g}$ of a white solid $\left(96.2 \%\right.$ yield). ${ }^{1} \mathrm{H} \mathrm{NMR}\left(\mathrm{CDCl}_{3}\right): \delta 2.59$ (broad $\mathrm{m}, 26,-$ $\mathrm{CH}_{2}-\mathrm{CH}_{2}-$ ), 2.69 (broad m, 52, - $\underline{\mathrm{CH}}_{2}-\mathrm{C}_{2}{ }^{-}$), 4.13 (m, 28, - $\underline{\mathrm{CH}}_{2}-\mathrm{CH}-\underline{\mathrm{CH}}_{2}-$ ), 4.13 (m, 28, $\left.\mathrm{C}_{2}-\mathrm{CH}-\underline{\mathrm{C}}_{2}-\right), 4.69\left(\mathrm{~m}, 8,-\mathrm{CH}_{2}-\mathrm{C} \underline{\mathrm{H}}-\mathrm{CH}_{2}-\right), 5.22\left(\mathrm{~m}, 6,-\mathrm{CH}_{2}-\mathrm{C} \underline{\mathrm{H}}-\mathrm{CH}_{2}-\right), 5.50(\mathrm{~s}, 8, \mathrm{C} \underline{\mathrm{H}})$, 7.32 (m, 24, arom. $\underline{\mathrm{H}}$ ), 7.47 (m, 16, arom. $\mathrm{C} \underline{\mathrm{H}}) .{ }^{13} \mathrm{C} \mathrm{NMR}\left(\mathrm{CDCl}_{3}\right): \delta 172.27$ (ㅁOOR),

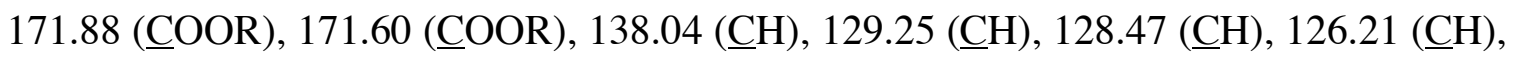
$101.30(\underline{\mathrm{CH}}), 69.48(\underline{\mathrm{CH}}), 69.15\left(\underline{\mathrm{CH}}_{2}\right), 66.54(\underline{\mathrm{CH}}), 62.57\left(\underline{\mathrm{CH}_{2}}\right), 29.35\left(\underline{\mathrm{C}} \mathrm{H}_{2}\right), 29.18$ $\left(\underline{\mathrm{CH}}_{2}\right) 29.03\left(\underline{\mathrm{CH}}_{2}\right), 28.84\left(\underline{\mathrm{CH}}_{2}\right)$. FTIR: $v\left(\mathrm{~cm}^{-1}\right) 2969$ (aliph. C-H stretch), $1733(\mathrm{C}=\mathrm{O})$. FAB MS $3060.7 \mathrm{~m} / \mathrm{z}\left(\mathrm{MH}^{+}\right)$(Theory: $3060.9 \mathrm{~m} / \mathrm{z}\left(\mathrm{M}^{+}\right)$). Elemental Analysis C: $59.20 \%$; H $5.64 \%$ (Theory C: $58.86 \%$; H $5.60 \%$ ). SEC $\mathrm{M}_{\mathrm{w}}: 3030, \mathrm{M}_{\mathrm{n}}: 2990$, PDI: $1.01 . \mathrm{T}_{\mathrm{g}}\left({ }^{\circ} \mathrm{C}\right.$ ): 42.3.

Synthesis of [G2]-PGLSA-OH (12) - Pd/C $(10 \%$ w/w $)$ was added to a solution of benzylidene protected [G2]-PGLSA $(2.04 \mathrm{~g}, 0.667 \mathrm{mmol})$ in THF $(20 \mathrm{~mL})$. The flask for catalytic hydrogenolysis was evacuated and filled with $50 \mathrm{psi}$ of $\mathrm{H}_{2}$ before shaking for 10 hours. The catalyst was filtered and washed with THF $(20 \mathrm{~mL})$. The filtrate was evaporated to give $1.49 \mathrm{~g}$ of a colorless, viscous oil (95\% yield). ${ }^{1} \mathrm{H}$ NMR $\left(\mathrm{CD}_{3} \mathrm{OD}\right): \delta$ $2.64\left(\mathrm{~m}, 52,-\mathrm{C}_{2}-\underline{\mathrm{CH}}_{2}-\right), 3.53$ (m, 16, - $\left.\mathrm{C}_{2}-\mathrm{CH}-\underline{\mathrm{CH}}_{2}-\right), 3.64$ (m, 4, - $\left.\mathrm{C}_{2}-\mathrm{CH}-\mathrm{C}_{2}-\right), 3.80$ 
(m, 8, - $\left.\mathrm{CH}_{2}-\mathrm{C} \underline{\mathrm{H}}-\mathrm{CH}_{2}-\right), 4.06$ (m, 8, $\left.-\mathrm{C}_{2}{ }_{2}-\mathrm{CH}-\mathrm{C}_{2}{ }^{-}\right), 4.14$ (m, 6, - $\left.\underline{\mathrm{CH}}_{2}-\mathrm{CH}-\mathrm{C}_{2}{ }^{-}\right), 4.21$ (m, 11, $\left.-\mathrm{CH}_{2}-\mathrm{CH}-\mathrm{CH}_{2}-\right), 4.30\left(\mathrm{~m}, 11,-\mathrm{CH}_{2}-\mathrm{CH}-\underline{\mathrm{CH}}_{2}-\right), 5.25$ (m, 6, - $\left.\mathrm{CH}_{2}-\mathrm{CH}-\mathrm{CH}_{2}-\right) .{ }^{13} \mathrm{C} \mathrm{NMR}$

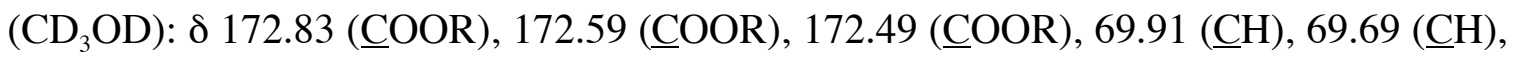
$65.68\left(\underline{\mathrm{C}} \mathrm{H}_{2}\right), 62.88\left(\underline{\mathrm{CH}}_{2}\right), 62.37\left(\underline{\mathrm{CH}}_{2}\right), 28.61\left(\underline{\mathrm{CH}}_{2}\right)$. FTIR: $v\left(\mathrm{~cm}^{-1}\right) 3429(\mathrm{OH}), 2952$ (aliph. C-H stretch), 1728 (C=O). MALDI MS $2357.3 \mathrm{~m} / \mathrm{z}\left(\mathrm{MH}^{+}\right)$(Theory: $2356.1 \mathrm{~m} / \mathrm{z}$ $\left(\mathrm{M}^{+}\right)$). Elemental Analysis C: $48.32 \%$; H $5.97 \%$ (Theory C: $47.92 \%$; H 5.90\%). SEC $\mathrm{M}_{\mathrm{w}}: 3060, \mathrm{M}_{\mathrm{n}}: 3000$, PDI: $1.02 . \mathrm{T}_{\mathrm{g}}\left({ }^{\circ} \mathrm{C}\right):-17.4$.

Synthesis of [G3]-PGLSA-bzld (13) - $\mathrm{Pd}(\mathrm{OH})_{2} / \mathrm{C}(20 \% \mathrm{w} / \mathrm{w})$ and activated carbon were added to a solution of [G2]-PGLSA-bzld (12.81 g, $4.22 \mathrm{mmol})$ in THF (100 mL). The flask for catalytic hydrogenolysis was evacuated and filled with 60 psi of $\mathrm{H}_{2}$ before shaking for 10 hours. The catalyst and activated carbon were filtered off and washed with THF (100 mL). From this solution, $1.822 \mathrm{~g}$ of [G2]-PGLSA-OH in THF was removed form the mixture. Next, 2-(cis-1,3-O-benzylidene glycerol)succinic acid mono ester anhydride $(45.91 \mathrm{~g}, 84.63 \mathrm{mmol})$ and then DMAP $(1.56 \mathrm{~g}, 12.76 \mathrm{mmol})$ were directly added to the deprotected core in the THF. The reaction was stirred at room temperature for 14 hours under nitrogen atmosphere. Any remaining anhydride was quenched by the addition of n-propanol $(8.0 \mathrm{~mL}, 88 \mathrm{mmol})$, which was allowed to stir for another 5 hours. The THF was removed under vacuum and the remaining contents were dissolved in DCM $(500 \mathrm{~mL})$ and washed once with $0.1 \mathrm{~N} \mathrm{HCl}(400 \mathrm{~mL})$ and three times with saturated sodium bicarbonate $(400 \mathrm{~mL})$. The organic phase was dried with $\mathrm{Na}_{2} \mathrm{SO}_{4}$, filtered, and concentrated before the dendrimer was precipitated in hexanes $(800 \mathrm{~mL})$ and cooled to $-20{ }^{\circ} \mathrm{C}$ overnight. The hexanes were decanted and the precipitate was isolated to yield $20.37 \mathrm{~g}$ of a white solid $\left(91.4 \%\right.$ yield). ${ }^{1} \mathrm{H}$ NMR $\left(\mathrm{CDCl}_{3}\right): \delta 2.57-2.72$ (broad 
m, 116, $\left.-\mathrm{C}_{2}{ }^{-} \mathrm{CH}_{2}-\right), 4.12\left(\mathrm{~m}, 60,-\mathrm{CH}_{2}-\mathrm{CH}-\underline{\mathrm{CH}}_{2}-\right), 4.23$ (m, 60, - $\left.\underline{\mathrm{CH}}_{2}-\mathrm{CH}-\mathrm{CH}_{2}-\right), 4.68$ (m, 16, - $\left.\mathrm{CH}_{2}-\mathrm{C} \underline{\mathrm{H}}-\mathrm{CH}_{2}-\right), 5.22\left(\mathrm{~m}, 14,-\mathrm{CH}_{2}-\mathrm{CH}-\mathrm{CH}_{2}-\right), 5.49$ (s, 16, $\mathrm{C}$ ), 7.33 (m, 48, arom. $\mathrm{C} \underline{\mathrm{H}}), 7.46$ (m, 32, arom. $\mathrm{C} \underline{\mathrm{H}}) .{ }^{13} \mathrm{C} \mathrm{NMR}\left(\mathrm{CDCl}_{3}\right): \delta 172.31$ (ㅁOOR), 171.97 (OOR), $171.65(\underline{\mathrm{COOR}}), 138.01(\underline{\mathrm{CH}}), 129.28(\mathrm{CH}), 128.49(\underline{\mathrm{CH}}), 126.21(\mathrm{CH}), 101.28(\mathrm{CH})$, $\left.69.45(\underline{\mathrm{CH}}), 69.16(\underline{\mathrm{CH}})_{2}\right), 66.53(\underline{\mathrm{CH}}), 62.59\left(\underline{\mathrm{CH}_{2}}\right), 29.32\left(\underline{\mathrm{CH}}_{2}\right), 29.16\left(\underline{\mathrm{CH}}_{2}\right) 29.01$ $\left(\underline{\mathrm{CH}}_{2}\right), 28.81\left(\underline{\mathrm{CH}}_{2}\right)$. FTIR: $v\left(\mathrm{~cm}^{-1}\right) 2984$ (aliph. C-H stretch), $1733(\mathrm{C}=\mathrm{O})$. MALDI MS $6553.4 \mathrm{~m} / \mathrm{z}\left(\mathrm{MH}^{+}\right)$(Theory: $6552.2 \mathrm{~m} / \mathrm{z}\left(\mathrm{M}^{+}\right)$). Elemental Analysis C: $58.50 \%$; H $5.66 \%$ (Theory C: $58.29 \%$; H $5.57 \%$ ). SEC $\mathrm{M}_{\mathrm{w}}: 5550, \mathrm{M}_{\mathrm{n}}: 5480$, PDI: 1.01. $\mathrm{T}_{\mathrm{g}}\left({ }^{\circ} \mathrm{C}\right): 45.7$.

Synthesis of [G3]-PGLSA-OH (14) - $\mathrm{Pd}(\mathrm{OH})_{2} / \mathrm{C}(20 \% \mathrm{w} / \mathrm{w})$ and activated carbon were added to a solution of [G3]-PGLSA-bzld (3.571 g, $8.071 \mathrm{mmol})$ in THF/MeOH (9:1) (25 $\mathrm{mL)}$. The flask for catalytic hydrogenolysis was evacuated and filled with $60 \mathrm{psi}$ of $\mathrm{H}_{2}$ before shaking for 10 hours. The catalyst and activated carbon were filtered off and washed with more of the THF/MeOH solution $(50 \mathrm{~mL})$ before the solvents were evaporated. The product was used directly in next reaction. ${ }^{1} \mathrm{H}$ NMR $\left(\mathrm{CD}_{3} \mathrm{OD}\right): \delta 2.64$ (m, 116, - $\left.\mathrm{C}_{2}-\underline{\mathrm{CH}}_{2}-\right), 3.51\left(\mathrm{~m}, 26,-\underline{\mathrm{CH}}_{2}-\mathrm{CH}-\underline{\mathrm{C}}_{2}{ }^{-}\right), 3.67$ (m, 28, - $\left.\underline{\mathrm{C}}_{2}-\mathrm{CH}-\underline{\mathrm{C}}_{2}-\right), 3.80$ (m, 12, - $\left.\mathrm{CH}_{2}-\mathrm{C} \underline{\mathrm{H}}-\mathrm{CH}_{2}-\right), 4.05$ (m, 14, - $\left.\underline{\mathrm{CH}}_{2}-\mathrm{CH}-\underline{\mathrm{C}}_{2}-\right), 4.14$ (m, 14, - $\underline{\mathrm{C}}_{2}-\mathrm{CH}-\underline{\mathrm{C}}_{2}-$ ), 4.22 (m, 22, - $\left.\mathrm{CH}_{2}-\mathrm{CH}-\mathrm{CH}_{2}-\right), 4.30\left(\mathrm{~m}, 22,-\mathrm{CH}_{2}-\mathrm{CH}-\mathrm{CH}_{2}-\right), 5.26\left(\mathrm{~m}, 14,-\mathrm{CH}_{2}-\mathrm{CH}-\mathrm{CH}_{2}\right) .{ }^{13} \mathrm{C}$ NMR $\left(\mathrm{CD}_{3} \mathrm{OD}\right): \delta 172.86(\underline{\mathrm{COOR}}), 69.91(\underline{\mathrm{CH}}), 67.64(\underline{\mathrm{CH}}), 65.67\left(\underline{\mathrm{CH}}_{2}\right), 62.87\left(\underline{\mathrm{CH}_{2}}\right)$, $62.41\left(\underline{\mathrm{CH}}_{2}\right), 28.61\left(\underline{\mathrm{C}}_{2}\right)$. FTIR: $v\left(\mathrm{~cm}^{-1}\right) 3442(\mathrm{OH}), 2959$ (aliph. C-H stretch), 1731 $(\mathrm{C}=\mathrm{O})$. MALDI MS $5144.8 \mathrm{~m} / \mathrm{z}\left(\mathrm{MH}^{+}\right)$(Theory: $5142.5 \mathrm{~m} / \mathrm{z}\left(\mathrm{M}^{+}\right)$). Elemental Analysis C: $48.07 \%$; H $5.84 \%$ (Theory C: $48.11 \%$; H $5.84 \%$ ). SEC $\mathrm{M}_{\mathrm{w}}:$ 5440, $\mathrm{M}_{\mathrm{n}}:$ 5370, PDI: 1.01. $\mathrm{T}_{\mathrm{g}}\left({ }^{\circ} \mathrm{C}\right):-15.5$. 
Synthesis of [G4]-PGLSA-bzld (15) - The deprotected core was dissolved in the THF/dimethyl acetimide (10:1) (200 mL) and 2-(cis-1,3-O-benzylidene glycerol)succinic acid mono ester anhydride $(60.83 \mathrm{~g}, 0.11 \mathrm{mmol})$ and then DMAP (1.63 g, $13.34 \mathrm{mmol})$ were directly added to the reaction flask. The reaction was stirred at room temperature for 14 hours under nitrogen atmosphere. Any remaining anhydride was quenched by the addition of n-propanol ( $4.0 \mathrm{~mL}, 44 \mathrm{mmol})$, which was allowed to stir for another 5 hours. The solvents were removed under vacuum and the remaining contents were dissolved in DCM $(250 \mathrm{~mL})$ and washed once with $0.1 \mathrm{~N} \mathrm{HCl}(200 \mathrm{~mL})$ and three times with saturated sodium bicarbonate $(200 \mathrm{~mL})$. The organic phase was dried with $\mathrm{Na}_{2} \mathrm{SO}_{4}$, filtered, and concentrated before the dendrimer was precipitated in hexanes $(450 \mathrm{~mL})$ and cooled to $-20{ }^{\circ} \mathrm{C}$ overnight. The hexanes were decanted and the precipitate was isolated to yield $33.25 \mathrm{~g}$ of a white solid (88.1\% yield). ). ${ }^{1} \mathrm{H}$ NMR $\left(\mathrm{CDCl}_{3}\right)$ : $\delta 2.63(\mathrm{~m}, 70$, $\left.\mathrm{C}_{2}-\mathrm{C}_{2}-\right), 2.72\left(\mathrm{~m}, 146,-\underline{\mathrm{CH}}_{2}-\underline{\mathrm{CH}}_{2}-\right), 2.90$ (m, 32, - $\left.\underline{\mathrm{C}}_{2}-\underline{\mathrm{C}}_{2}-\right), 4.14$ (m, 100, - $\underline{\mathrm{C}}_{2}-\mathrm{CH}-$ $\left.\mathrm{C}_{2}{ }^{-}\right), 4.25\left(\mathrm{~m}, 100,-\underline{\mathrm{C}}_{2}-\mathrm{CH}-\mathrm{C}_{2}{ }^{-}\right), 4.70\left(\mathrm{~m}, 32,-\mathrm{CH}_{2}-\mathrm{C} \underline{\mathrm{H}}-\mathrm{CH}_{2}-\right), 5.25\left(\mathrm{~m}, 16,-\mathrm{CH}_{2^{-}}\right.$ C$\left.-\mathrm{CH}_{2}-\right), 5.52$ (s, 32, $\left.\mathrm{C} \underline{\mathrm{H}}\right), 7.33$ (m, 96, arom. $\left.\mathrm{C} \underline{\mathrm{H}}\right), 7.47$ (m, 64, arom. $\left.\mathrm{C} \underline{\mathrm{H}}\right) .{ }^{13} \mathrm{C} \mathrm{NMR}$

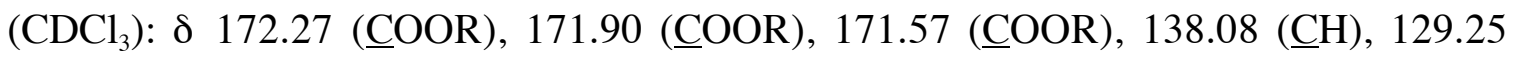
$(\underline{\mathrm{C} H}), 128.47(\underline{\mathrm{CH}}), 126.23(\underline{\mathrm{CH}}), 101.27(\underline{\mathrm{CH}}), 69.49(\underline{\mathrm{CH}}), 69.13\left(\underline{\mathrm{CH}}_{2}\right), 66.54(\underline{\mathrm{CH}})$, $62.45\left(\underline{\mathrm{CH}}_{2}\right), 29.34\left(\underline{\mathrm{CH}}_{2}\right), 29.02\left(\underline{\mathrm{CH}}_{2}\right), 28.83\left(\underline{\mathrm{CH}}_{2}\right)$. FTIR: $v\left(\mathrm{~cm}^{-1}\right) 2978$ (aliph. C-H stretch), $1733(\mathrm{C}=\mathrm{O})$. MALDI MS $13536.8 \mathrm{~m} / \mathrm{z}\left(\mathrm{MH}^{+}\right)$(Theory: $\left.13534.7 \mathrm{~m} / \mathrm{z}\left(\mathrm{M}^{+}\right)\right)$. Elemental Analysis C: $58.20 \%$; H $5.56 \%$ (Theory C: $58.04 \%$; H $5.56 \%$ ). SEC $\mathrm{M}_{\mathrm{w}}: 9000, \mathrm{M}_{\mathrm{n}}: 8900$, PDI: $1.01 . \mathrm{T}_{\mathrm{g}}\left({ }^{\circ} \mathrm{C}\right): 47.0$.

Synthesis of [G4]-PGLSA-OH (16) - Pd/C (10\% w/w) was added to a solution of benzylidene protected [G4]-PGLSA $(0.477 \mathrm{~g}, 0.0352 \mathrm{mmol})$ in 9:1 THF/MeOH $(20 \mathrm{~mL})$. 
The flask for catalytic hydrogenolysis was evacuated and filled with $50 \mathrm{psi}$ of $\mathrm{H}_{2}$ before shaking for 10 hours. The catalyst was filtered and washed with 9:1 THF/MeOH (20 $\mathrm{mL}$ ). The filtrate was evaporated to give $0.351 \mathrm{~g}$ of a colorless, viscous oil (93\% yield). ${ }^{1} \mathrm{H}$ NMR (CD 3 OD): $\delta 2.65$ (m, 244, - $\left.\mathrm{C}_{2}-\mathrm{C}_{2}-\right), 3.53$ (m, 50, - $\left.\underline{\mathrm{CH}}_{2}-\mathrm{CH}-\mathrm{C} \underline{\mathrm{H}}_{2}\right), 3.65$ (m, 22, - $\left.\mathrm{CH}_{2}-\mathrm{CH}-\mathrm{CH}_{2}-\right), 3.81\left(\mathrm{~m}, 28,-\mathrm{CH}_{2}-\mathrm{C} \underline{H}-\mathrm{CH}_{2}-\right), 4.05\left(\mathrm{~m}, 32,-\underline{\mathrm{C}}_{2}-\mathrm{CH}-\mathrm{C}_{2}-\right), 4.14(\mathrm{~m}$, 32, - $\left.\underline{\mathrm{CH}}_{2}-\mathrm{CH}-\underline{\mathrm{C}}_{2}{ }^{-}\right), 4.24$ (m, 60, - $\left.\mathrm{C}_{2}-\mathrm{CH}-\underline{\mathrm{H}}_{2}{ }^{-}\right), 4.30$ (m, 60, - $\left.\underline{\mathrm{C}}_{2}-\mathrm{CH}-\underline{\mathrm{CH}}_{2}-\right), 5.26$ (m, 32, $\left.-\mathrm{CH}_{2}-\mathrm{C} \underline{\mathrm{H}}-\mathrm{CH}_{2}-\right) .{ }^{13} \mathrm{C}$ NMR $\left(\mathrm{CD}_{3} \mathrm{OD}\right): \delta 172.94(\underline{\mathrm{COOR}}), 69.92(\underline{\mathrm{C}} \mathrm{H}), 65.72\left(\underline{\mathrm{C}} \mathrm{H}_{2}\right)$, $62.91\left(\underline{\mathrm{CH}}_{2}\right), 28.67\left(\underline{\mathrm{CH}}_{2}\right)$. FTIR: $v\left(\mathrm{~cm}^{-1}\right) 3444(\mathrm{OH}), 2931$ (aliph. C-H stretch), 1729 $(\mathrm{C}=\mathrm{O})$. MALDI MS $10715.6 \mathrm{~m} / \mathrm{z}\left(\mathrm{MH}^{+}\right)\left(\right.$Theory: $\left.10715.3 \mathrm{~m} / \mathrm{z}\left(\mathrm{M}^{+}\right)\right)$. Elemental Analysis C: $48.50 \%$; H $5.83 \%$ (Theory C: $48.20 \%$; H $5.81 \%$ ). SEC $\mathrm{M}_{\mathrm{w}}:$ 8800, $\mathrm{M}_{\mathrm{n}}$ : 8720, PDI: $1.01 . \mathrm{T}_{\mathrm{g}}\left({ }^{\circ} \mathrm{C}\right):-13.5$.

Synthesis of [G0]-PGLAA-bzld (17) - Adipic acid (6.47 g, $44.30 \mathrm{mmol})$, cis-1,3-Obenzylideneglycerol (17.57 g, $97.51 \mathrm{mmol})$, and DPTS (10.01 g, $34.03 \mathrm{mmol})$ were dissolved in DCM $(120 \mathrm{~mL})$ followed by the addition of DCC (28.26 g, $136.96 \mathrm{mmol})$. The reaction was stirred at room temperature for 14 hours under nitrogen atmosphere. Upon reaction completion, the DCC-urea was filtered and washed with a small amount of DCM $(50 \mathrm{~mL})$. The crude product was purified by silica gel chromatography, eluting with $2 \% \mathrm{MeOH}$ in DCM. The appropriate isolated fractions were concentrated, filtered (to remove any DCU), and directly precipitated in hexanes and cooled to $-20{ }^{\circ} \mathrm{C}$ overnight. Following vacuum filtration, $12.69 \mathrm{~g}$ of a white solid was collected $(60.8 \%$ yield). ${ }^{1} \mathrm{H}$ NMR (400 MHz, $\mathrm{CDCl}_{3}$ ): $\delta 1.72$ (s, 4, - $\mathrm{CH}_{2}-\mathrm{C}_{2}-\underline{\mathrm{C}}_{2}-\mathrm{CH}_{2}-$ ), 2.45 (s, 4, - $\mathrm{C}_{2^{-}}$ $\left.\mathrm{CH}_{2}-\mathrm{CH}_{2}-\underline{\mathrm{CH}}_{2}-\right), 4.12\left(\mathrm{~m}, 4,-\underline{\mathrm{C}}_{2}-\mathrm{CH}-\mathrm{C}_{2}-\right), 4.25$ (m, 4, - $\left.\underline{\mathrm{C}}_{2}-\mathrm{CH}-\mathrm{C} \underline{\mathrm{H}}_{2}-\right), 4.68$ (m, 2, $\left.\mathrm{CH}_{2}-\mathrm{C} \underline{\mathrm{H}}-\mathrm{CH}_{2}-\right), 5.52$ (s, 2, $\left.\mathrm{C} \underline{\mathrm{H}}\right), 7.34$ (m, 6, arom. $\left.\mathrm{C} \underline{\mathrm{H}}\right), 7.48$ (m, 4, arom. $\left.\mathrm{C} \underline{\mathrm{H}}\right) .{ }^{13} \mathrm{C} \mathrm{NMR}$ 
$\left(100.6 \mathrm{MHz}, \mathrm{CDCl}_{3}\right): \delta 173.47(\underline{\mathrm{COOR}}), 138.01(\underline{\mathrm{CH}}), 129.27(\underline{\mathrm{C}} \mathrm{H}), 128.50(\underline{\mathrm{CH}}), 126.22$ $\left.(\underline{\mathrm{C}} \mathrm{H}), 101.43(\underline{\mathrm{C}} \mathrm{H}), 69.30\left(\underline{\mathrm{CH}}_{2}\right), 66.08(\underline{\mathrm{C}} \mathrm{H}), 34.15\left(\underline{\mathrm{CH}}_{2}\right), 24.49(\underline{\mathrm{CH}})_{2}\right)$. FTIR: v $\left(\mathrm{cm}^{-1}\right)$ 2930 and 2862 (aliph. C-H stretch), 1714 (C=O). FAB $471.2 \mathrm{~m} / \mathrm{z}[\mathrm{M}+\mathrm{H}]^{+}$(Theory: 470.5 m/z [M] ${ }^{+}$). Elemental Analysis C: $66.32 \%$; H $6.50 \%$ (Theory C: $66.37 \%$; H $6.43 \%$ ). SEC $M_{\mathrm{w}}: 410, \mathrm{M}_{\mathrm{n}}: 410$, PDI: $1.00 . \mathrm{T}_{\mathrm{m}}\left({ }^{\circ} \mathrm{C}\right): 1458$.

Synthesis of [G0]-PGLAA-OH (18) - $\mathrm{Pd}(\mathrm{OH})_{2} / \mathrm{C}(10 \% \mathrm{w} / \mathrm{w})$ was added to a solution of [G0]-PGLAA-bzld $(2.16 \mathrm{~g}, 4.59 \mathrm{mmol})$ in THF $(30 \mathrm{~mL})$. The flask for catalytic hydrogenolysis was evacuated and filled with 60 psi of $\mathrm{H}_{2}$ before shaking for 10 hours. The catalyst was filtered and washed with THF solution $(50 \mathrm{~mL})$. The filtrate was evaporated to give $1.303 \mathrm{~g}$ of a clear viscous oil (96.4\% yield). ${ }^{1} \mathrm{H}$ NMR (400 MHz, $\left.\mathrm{CD}_{3} \mathrm{OD}\right): \delta 1.64\left(\mathrm{~m}, 4,-\mathrm{CH}_{2}-\mathrm{CH}_{2}-\mathrm{C}_{2}-\mathrm{CH}_{2}-\right), 2.36\left(\mathrm{~m}, 4,-\mathrm{C}_{2}-\mathrm{CH}_{2}-\mathrm{CH}_{2}-\mathrm{CH}_{2}-\right), 3.51(\mathrm{~m}$, 1, - $\left.\underline{\mathrm{C}}_{2}-\mathrm{C} \underline{\mathrm{H}}-\mathrm{C}_{2}{ }^{-}\right), 3.64\left(\mathrm{~m}, 5,-\mathrm{C}_{2}-\mathrm{C} \underline{\mathrm{H}}-\mathrm{C}_{2}{ }^{-}\right), 3.78$ (m, 1, - $\left.\underline{\mathrm{C}}_{2}-\mathrm{C} \underline{\mathrm{H}}-\mathrm{C}_{2}-\right), 4.03$ (m, 1, $\left.\mathrm{C}_{2}-\mathrm{C} \underline{\mathrm{H}}-\underline{\mathrm{C}}_{2}-\right), 4.12$ (m, 1, - $\left.\underline{\mathrm{CH}}_{2}-\mathrm{C} \underline{\mathrm{H}}-\underline{\mathrm{C}}_{2}-\right) .{ }^{13} \mathrm{C} \mathrm{NMR}\left(100.6 \mathrm{MHz}, \mathrm{CD}_{3} \mathrm{OD}\right): \delta 173.76$ (ㅁOR), $75.43(\underline{\mathrm{CH}}), 69.91(\underline{\mathrm{C}} \mathrm{H}), 65.33\left(\underline{\mathrm{C}} \mathrm{H}_{2}\right), 62.83\left(\underline{\mathrm{CH}}_{2}\right), 60.49\left(\underline{\mathrm{CH}}_{2}\right), 33.52\left(\underline{\mathrm{C}} \mathrm{H}_{2}\right)$, $33.31\left(\underline{\mathrm{CH}}_{2}\right), 24.12\left(\underline{\mathrm{CH}}_{2}\right)$. FTIR: $v\left(\mathrm{~cm}^{-1}\right) 3389(\mathrm{OH}), 2944$ (aliph. C-H stretch), 1720 $(\mathrm{C}=\mathrm{O})$. FAB MS $295.3 \mathrm{~m} / \mathrm{z}[\mathrm{M}+\mathrm{H}]^{+}$(Theory: $294.3 \mathrm{~m} / \mathrm{z}[\mathrm{M}]^{+}$). Elemental Analysis C: $48.44 \%$; H $7.65 \%$ (Theory C: $48.97 \%$; H $7.53 \%$ ). SEC $M_{\mathrm{w}}:$ 470, $\mathrm{M}_{\mathrm{n}}: 460$, PDI: 1.01. Synthesis of [G1]-PGLAA-bzld (19) - 2-(cis-1,3-O-benzylidene glycerol)adipic acid mono ester, 6, (7.23 g, $23.43 \mathrm{mmol})$, [G0]-PGLAA-OH (1.22 g, $4.15 \mathrm{mmol})$, and DPTS (2.83 g, $9.62 \mathrm{mmol})$ were dissolved in THF $(100 \mathrm{~mL})$ followed by the addition of DCC (4.32 $\mathrm{g}, 21.0 \mathrm{mmol})$. The reaction was stirred at room temperature for 14 hours under nitrogen atmosphere. Upon reaction completion, the DCU was filtered and washed with a 
small amount of THF $(50 \mathrm{~mL})$. The crude product was purified by silica gel chromatography, eluting with 1:1 to 4:1 EtOAc:hexanes. The appropriate isolated fractions were concentrated, filtered (to remove any DCU), and directly precipitated in hexanes and cooled to $-20{ }^{\circ} \mathrm{C}$ overnight. The hexanes were decanted and the precipitate was isolated to yield $5.99 \mathrm{~g}$ of a sticky solid $\left(99.1 \%\right.$ yield). ${ }^{1} \mathrm{H}$ NMR (400 $\left.\mathrm{MHz}, \mathrm{CDCl}_{3}\right)$ :

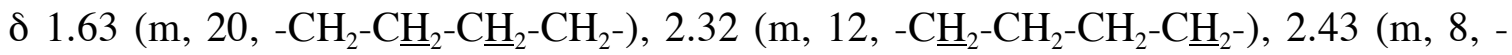
$\left.\mathrm{C}_{2}-\mathrm{CH}_{2}-\mathrm{CH}_{2}-\underline{\mathrm{CH}}_{2}-\right), 4.10\left(\mathrm{~m}, 12,-\mathrm{C}_{2}-\mathrm{CH}-\mathrm{C}_{2}-\right), 4.25\left(\mathrm{~m}, 12,-\underline{\mathrm{CH}}_{2}-\mathrm{CH}-\underline{\mathrm{C}}_{2}-\right), 4.68$ (m, 4, - $\mathrm{CH}_{2}-\mathrm{CH}-\mathrm{CH}_{2}-$ ), 5.21 (m, 2, - $\mathrm{CH}_{2}-\mathrm{C} \underline{\mathrm{H}}-\mathrm{CH}_{2}-$ ), 5.51 (s, 4, $\mathrm{C}$ ), 7.32 (m, 12, arom. $\mathrm{C} \underline{\mathrm{H}}), 7.47$ (m, 8, arom. $\mathrm{C} \underline{\mathrm{H}}) .{ }^{13} \mathrm{C} \mathrm{NMR}\left(100.6 \mathrm{MHz}, \mathrm{CDCl}_{3}\right): \delta 173.40$ (OOR), 172.87 (COOR), $172.55(\underline{\mathrm{COOR}}), 138.02(\underline{\mathrm{CH}}), 129.28(\underline{\mathrm{CH}}), 128.49(\underline{\mathrm{CH}}), 126.21(\underline{\mathrm{CH}}), 101.39$ $(\underline{\mathrm{C}} \mathrm{H}), 69.28\left(\underline{\mathrm{CH}}_{2}\right), 66.11(\underline{\mathrm{CH}}), 62.39\left(\underline{\mathrm{C}} \mathrm{H}_{2}\right), 34.08\left(\underline{\mathrm{C}} \mathrm{H}_{2}\right), 33.90\left(\underline{\mathrm{CH}}_{2}\right), 33.75\left(\underline{\mathrm{C}} \mathrm{H}_{2}\right)$, $24.37\left(\mathrm{CH}_{2}\right)$. FTIR: $v\left(\mathrm{~cm}^{-1}\right) 2948$ and 2863 (aliph. C-H stretch), $1730(\mathrm{C}=\mathrm{O})$. FAB MS $1455.6 \mathrm{~m} / \mathrm{z}[\mathrm{M}+\mathrm{H}]^{+}$(Theory: $1455.5 \mathrm{~m} / \mathrm{z}[\mathrm{M}]^{+}$). Elemental Analysis C: $62.22 \%$; H 6.51 $\%$ (Theory C: $62.71 \% ; \mathrm{H} 6.51 \%$ ). SEC $\mathrm{M}_{\mathrm{w}}: 1560, \mathrm{M}_{\mathrm{n}}: 1530$, PDI: $1.02 . \mathrm{T}_{\mathrm{g}}\left({ }^{\circ} \mathrm{C}\right):-0.6$.

Synthesis of [G1]-PGLAA-OH (20) - $\mathrm{Pd}(\mathrm{OH})_{2} / \mathrm{C}(20 \%$ w/w) was added to a solution of [G1]-PGLAA-bzld $(4.87 \mathrm{~g}, 3.35 \mathrm{mmol})$ in THF $(50 \mathrm{~mL})$. The flask for catalytic hydrogenolysis was evacuated and filled with $60 \mathrm{psi}$ of $\mathrm{H}_{2}$ before shaking for 10 hours. The catalyst was filtered and washed with THF solution $(50 \mathrm{~mL})$. The filtrate was evaporated to give $3.67 \mathrm{~g}$ of a clear viscous oil (99.5\% yield). ${ }^{1} \mathrm{H}$ NMR (400 MHz, $\left.\mathrm{CD}_{3} \mathrm{OD}\right): \delta 1.63\left(\mathrm{~m}, 20,-\mathrm{CH}_{2}-\underline{\mathrm{C}}_{2}-\mathrm{C}_{2}-\mathrm{CH}_{2}-\right), 2.36\left(\mathrm{~m}, 20,-\mathrm{CH}_{2}-\mathrm{CH}_{2}-\mathrm{CH}_{2}-\mathrm{CH}_{2}-\right), 3.52$ (m, 2, - $\mathrm{C}_{2}-\mathrm{C} \underline{\mathrm{H}}-\mathrm{C}_{2^{-}}-$), 3.59-3.69 (broad m, 12, - $\underline{\mathrm{CH}}_{2}-\mathrm{C} \underline{\mathrm{H}}-\mathrm{C}_{2_{2}}$ ), 3.79 (m, 1, - $\underline{\mathrm{CH}}_{2}-\mathrm{C} \underline{\mathrm{H}}-$

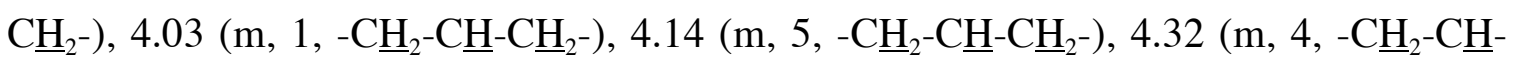
$\mathrm{CH}_{2}-$ ), 5.24 (m, 2, - $\left.\mathrm{CH}_{2}-\mathrm{C} \underline{H}-\mathrm{CH}_{2}-\right) .{ }^{13} \mathrm{C} \mathrm{NMR}\left(100.6 \mathrm{MHz}, \mathrm{CD}_{3} \mathrm{OD}\right): \delta 173.64$ (COOR), 


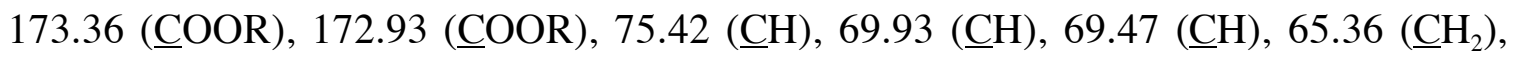
$62.87\left(\underline{\mathrm{C}} \mathrm{H}_{2}\right), 62.15(\underline{\mathrm{C}} \mathrm{H}), 60.50\left(\underline{\mathrm{CH}}_{2}\right), 33.49\left(\underline{\mathrm{CH}}_{2}\right), 33.35\left(\underline{\mathrm{CH}}_{2}\right), 33.20\left(\underline{\mathrm{CH}}_{2}\right), 24.11$ $\left(\mathrm{CH}_{2}\right)$. FTIR: $v\left(\mathrm{~cm}^{-1}\right) 3432(\mathrm{OH}), 2943$ (aliph. C-H stretch), $1728(\mathrm{C}=\mathrm{O})$. MALDI-TOF MS 1126 m/z [M+Na] $]^{+}$(Theory: 1103 m/z [M] ${ }^{+}$). Elemental Analysis C: $52.12 \%$; H 7.35 \% (Theory C: $52.26 \%$; H $7.13 \%$ ). SEC $\mathrm{M}_{\mathrm{w}}: 1660, \mathrm{M}_{\mathrm{n}}: 1630$, PDI: 1.01. $\mathrm{T}_{\mathrm{g}}\left({ }^{\circ} \mathrm{C}\right):-35.2$.

Synthesis of [G2]-PGLAA-bzld (21) - 2-(cis-1,3-O-benzylidene glycerol)adipic acid mono ester (10.01 g, $32.47 \mathrm{mmol})$, [G1]-PGLAA-OH (3.40 g, $3.08 \mathrm{mmol})$, and DPTS $(2.51 \mathrm{~g}, 8.53 \mathrm{mmol})$ were dissolved in THF $(100 \mathrm{~mL})$ followed by the addition of DCC (4.62 $\mathrm{g}, 22.4 \mathrm{mmol})$. The reaction was stirred at room temperature for 14 hours under nitrogen atmosphere. Upon reaction completion, the DCU was filtered and washed with a small amount of THF $(50 \mathrm{~mL})$. The crude product was purified by silica gel chromatography, eluting with $2 \% \mathrm{MeOH}$ in DCM. The appropriate isolated fractions were concentrated, filtered (to remove any DCU), and directly precipitated in hexanes and cooled to $-20{ }^{\circ} \mathrm{C}$ overnight. The hexanes were decanted and the precipitate was isolated to yield $9.39 \mathrm{~g}$ of a sticky wax (89.0\% yield). ${ }^{1} \mathrm{H} \mathrm{NMR}\left(400 \mathrm{MHz}, \mathrm{CDCl}_{3}\right): \delta$ $1.63\left(\mathrm{~m}, 52,-\mathrm{CH}_{2}-\underline{\mathrm{C}}_{2}-\mathrm{C}_{2}-\mathrm{CH}_{2}-\right), 2.31\left(\mathrm{~m}, 36,-\underline{\mathrm{C}}_{2}-\mathrm{CH}_{2}-\mathrm{CH}_{2}-\underline{\mathrm{C}}_{2}-\right), 2.41$ (m, 16, $\left.\mathrm{CH}_{2}-\mathrm{CH}_{2}-\mathrm{CH}_{2}-\mathrm{CH}_{2}{ }^{-}\right), 4.05$ (m, 28, - $\left.\mathrm{C}_{2}-\mathrm{CH}-\mathrm{C}_{2}{ }^{-}\right), 4.25$ (m, 28, $\left.-\underline{\mathrm{CH}}_{2}-\mathrm{CH}-\mathrm{CH}_{2}-\right), 4.67$ (m, 8, - $\left.\mathrm{CH}_{2}-\mathrm{C} \underline{\mathrm{H}}-\mathrm{CH}_{2}-\right), 5.21$ (m, 6, - $\mathrm{CH}_{2}-\mathrm{C} \underline{\mathrm{H}}-\mathrm{CH}_{2}-$ ), 5.51 (s, 8, $\mathrm{C}$ ), 7.33 (m, 24, arom. $\mathrm{C} \underline{\mathrm{H}}), 7.46$ (m, 16, arom. $\mathrm{C} \underline{\mathrm{H}}) .{ }^{13} \mathrm{C}$ NMR (100.6 MHz, $\left.\mathrm{CDCl}_{3}\right): \delta 173.39$ (OOR), 172.87 (OOR), 172.54 (요), $138.02(\underline{\mathrm{CH}}), 129.27(\underline{\mathrm{CH}}), 128.49(\underline{\mathrm{C}} \mathrm{H}), 126.21(\underline{\mathrm{CH}}), 101.38$ $(\underline{\mathrm{CH}}), 69.27\left(\underline{\mathrm{CH}}_{2}\right), 66.11(\underline{\mathrm{CH}}), 62.39\left(\underline{\mathrm{CH}}_{2}\right), 34.08\left(\mathrm{CH}_{2}\right), 33.74\left(\underline{\mathrm{CH}}_{2}\right), 33.67\left(\mathrm{CH}_{2}\right)$, $24.37\left(\underline{\mathrm{CH}}_{2}\right)$. FTIR: $v\left(\mathrm{~cm}^{-1}\right) 2945$ and 2863 (aliph. C-H stretch), $1731(\mathrm{C}=\mathrm{O})$. MALDI 
MS 3449 m/z [M+Na] $]^{+}$(Theory: 3426 m/z [M] ${ }^{+}$). Elemental Analysis C: $61.66 \%$; H 6.57 $\%$ (Theory C: $61.71 \%$; H $6.53 \%$ ). SEC $\mathrm{M}_{\mathrm{w}}: 3810, \mathrm{M}_{\mathrm{n}}: 3760$, PDI: $1.01 . \mathrm{T}_{\mathrm{g}}\left({ }^{\circ} \mathrm{C}\right): 4.4$.

Synthesis of [G2]-PGLAA-OH (22) - $\mathrm{Pd}(\mathrm{OH})_{2} / \mathrm{C}(20 \% \mathrm{w} / \mathrm{w})$ was added to a solution of [G2]-PGLAA-bzld (8.02 g, $2.34 \mathrm{mmol})$ in THF (100 mL). The flask for catalytic hydrogenolysis was evacuated and filled with 60 psi of $\mathrm{H}_{2}$ before shaking for 10 hours. The catalyst was filtered and washed with THF solution $(50 \mathrm{~mL})$. The filtrate was evaporated to give $6.36 \mathrm{~g}$ of a clear viscous oil (99.4\% yield). ${ }^{1} \mathrm{H}$ NMR (400 MHz, $\left.\mathrm{CD}_{3} \mathrm{OD}\right): \delta 1.62\left(\mathrm{~m}, 52,-\mathrm{CH}_{2}-\mathrm{C}_{2}-\mathrm{C}_{2}-\mathrm{CH}_{2}-\right), 2.35\left(\mathrm{~m}, 52,-\underline{\mathrm{H}}_{2}-\mathrm{CH}_{2}-\mathrm{CH}_{2}-\mathrm{C}_{2}-\right), 3.52$ (m, 5, - $\underline{\mathrm{C}}_{2}-\mathrm{C} \underline{\mathrm{H}}-\mathrm{C} \underline{\mathrm{H}}_{2}-$ ), 3.59-3.71 (broad m, 25, - $\underline{\mathrm{C}}_{2}-\mathrm{C} \underline{\mathrm{H}}-\mathrm{C}_{2}{ }_{2}$ ), 3.79 (m, 3, - $\underline{\mathrm{C}}_{2}-\mathrm{C} \underline{\mathrm{H}}-$ $\left.\mathrm{C}_{2}-\right), 4.03$ (m, 3, - $\left.\underline{\mathrm{C}}_{2}-\mathrm{C} \underline{\mathrm{H}}-\mathrm{C} \underline{\mathrm{H}}_{2}-\right), 4.14$ (m, 15, - $\left.\mathrm{C}_{2}-\mathrm{C} \underline{\mathrm{H}}-\underline{\mathrm{C}}_{2}-\right), 4.33$ (m, 12, - $\underline{\mathrm{C}}_{2}-\mathrm{C} \underline{\mathrm{H}}-$ $\left.\mathrm{C}_{2}{ }^{-}\right), 5.25$ (m, 6, - $\left.\mathrm{CH}_{2}-\mathrm{C} \underline{\mathrm{H}}-\mathrm{CH}_{2}-\right) .{ }^{13} \mathrm{C} \mathrm{NMR}\left(100.6 \mathrm{MHz}, \mathrm{CD}_{3} \mathrm{OD}\right): \delta 173.63$ (ㅁO), 173.27 (ㅁOR), 172.92 (ㅁO), $75.42(\underline{\mathrm{CH}}), 69.94(\underline{\mathrm{CH}}), 69.47(\underline{\mathrm{CH}}), 65.38\left(\underline{\mathrm{C}} \mathrm{H}_{2}\right)$, $62.89\left(\underline{\mathrm{CH}}_{2}\right), 62.17\left(\underline{\mathrm{CH}}_{2}\right), 60.52\left(\underline{\mathrm{CH}}_{2}\right), 33.51\left(\underline{\mathrm{CH}}_{2}\right), 33.39\left(\underline{\mathrm{CH}}_{2}\right), 33.22\left(\underline{\mathrm{C}} \mathrm{H}_{2}\right), 24.12$ $\left(\underline{\mathrm{CH}}_{2}\right)$. FTIR: $v\left(\mathrm{~cm}^{-1}\right) 3460(\mathrm{OH}), 2945$ (aliph. C-H stretch), $1729(\mathrm{C}=\mathrm{O})$. MALDI-TOF MS $2744 \mathrm{~m} / \mathrm{z}[\mathrm{M}+\mathrm{Na}]^{+}$(Theory: $2721 \mathrm{~m} / \mathrm{z}[\mathrm{M}]^{+}$). Elemental Analysis C: $53.16 \%$; H 7.02 \% (Theory C: $52.97 \%$; H $7.04 \%$ ). SEC $\mathrm{M}_{\mathrm{w}}: 3690, \mathrm{M}_{\mathrm{n}}: 3630$, PDI: $1.02 . \mathrm{T}_{\mathrm{g}}\left({ }^{\circ} \mathrm{C}\right):-30.8$. Synthesis of [G3]-PGLAA-bzld (23) - 2-(cis-1,3-O-benzylidene glycerol)adipic acid mono ester (12.63 g, $40.95 \mathrm{mmol})$, [G2]-PGLAA-OH (5.26 g, $1.93 \mathrm{mmol}$ ), and DPTS (3.23 g, $10.99 \mathrm{mmol})$ were dissolved in THF $(100 \mathrm{~mL})$ followed by the addition of DCC (12.58 g, $60.97 \mathrm{mmol})$. The reaction was stirred at room temperature for 14 hours under nitrogen atmosphere. Upon reaction completion, the DCU was filtered and washed with a small amount of THF $(60 \mathrm{~mL})$. The crude product was purified by silica gel 
chromatography, eluting with 1.5 to $3.0 \% \mathrm{MeOH}$ in DCM. The appropriate isolated fractions were concentrated, filtered (to remove any DCU), and directly precipitated in hexanes and cooled to $-20{ }^{\circ} \mathrm{C}$ overnight. The hexanes were decanted and the precipitate was isolated to yield $12.22 \mathrm{~g}$ of a sticky wax (85.8 \% yield). ${ }^{1} \mathrm{H}$ NMR (400 MHz, $\mathrm{CDCl}_{3}$ ): $\delta 1.63$ (broad m, 130, $\left.-\mathrm{CH}_{2}-\mathrm{C}_{2}-\mathrm{C}_{2}-\mathrm{CH}_{2}-\right), 2.31$ (m, 90, - $\mathrm{C}_{2}-\mathrm{CH}_{2}-\mathrm{CH}_{2}-\mathrm{C}_{2}-$ ), $2.41\left(\mathrm{~m}, 32,-\mathrm{C}_{2}-\mathrm{CH}_{2}-\mathrm{CH}_{2}-\underline{\mathrm{CH}}_{2}-\right), 4.10\left(\mathrm{~m}, 62,-\underline{\mathrm{CH}}_{2}-\mathrm{CH}-\underline{\mathrm{CH}}_{2}-\right), 4.24$ (m, 62, - $\underline{\mathrm{C}}_{2}-\mathrm{CH}-$ $\left.\mathrm{C}_{2}{ }^{-}\right), 4.67$ (m, 16, - $\left.\mathrm{CH}_{2}-\mathrm{C} \underline{\mathrm{H}}-\mathrm{CH}_{2}-\right), 5.19\left(\mathrm{~m}, 14,-\mathrm{CH}_{2}-\mathrm{C} \underline{\mathrm{H}}-\mathrm{CH}_{2}-\right), 5.51$ (s, 16, $\left.\mathrm{C} \underline{\mathrm{H}}\right), 7.32$ (m, 48, arom. $\mathrm{CH}), 7.46$ (m, 32, arom. $\mathrm{C} \underline{\mathrm{H}}) .{ }^{13} \mathrm{C} \mathrm{NMR}\left(100.6 \mathrm{MHz}, \mathrm{CDCl}_{3}\right): \delta 173.38$

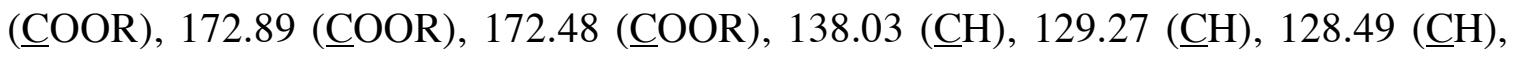
$126.21(\underline{\mathrm{CH}}), 101.36(\underline{\mathrm{CH}}), 69.26\left(\underline{\mathrm{CH}}_{2}\right), 66.11(\underline{\mathrm{CH}}), 62.29\left(\mathrm{CH}_{2}\right), 34.08\left(\mathrm{CH}_{2}\right), 33.83$ $\left(\underline{\mathrm{CH}}_{2}\right), 33.74\left(\underline{\mathrm{CH}}_{2}\right), 33.67\left(\underline{\mathrm{CH}}_{2}\right), 24.43\left(\underline{\mathrm{CH}}_{2}\right), 24.36\left(\underline{\mathrm{CH}}_{2}\right)$. FTIR: v $\left(\mathrm{cm}^{-1}\right) 2947$ (aliph. C-H stretch), $1731(\mathrm{C}=\mathrm{O})$. MALDI-TOF MS $7390 \mathrm{~m} / \mathrm{z}[\mathrm{M}+\mathrm{Na}]^{+}$(Theory: $7366 \mathrm{~m} / \mathrm{z}$ $[\mathrm{M}]^{+}$). Elemental Analysis C: $61.08 \%$; H $6.67 \%$ (Theory C: $61.31 \%$; H $6.54 \%$ ). SEC $\mathrm{M}_{\mathrm{w}}: 7230, \mathrm{M}_{\mathrm{n}}: 7130$, PDI: $1.02 . \mathrm{T}_{\mathrm{g}}\left({ }^{\circ} \mathrm{C}\right): 2.5$.

Synthesis of [G3]-PGLAA-OH (24) - $\mathrm{Pd}(\mathrm{OH})_{2} / \mathrm{C}(20 \% \mathrm{w} / \mathrm{w})$ was added to a solution of [G3]-PGLAA-bzld $(11.03 \mathrm{~g}, 1.50 \mathrm{mmol})$ in THF $(125 \mathrm{~mL})$. The flask for catalytic hydrogenolysis was evacuated and filled with $60 \mathrm{psi}$ of $\mathrm{H}_{2}$ before shaking for 10 hours. The catalyst was filtered and washed with THF solution $(75 \mathrm{~mL})$. The filtrate was evaporated to give $8.69 \mathrm{~g}$ of a clear viscous oil (97.5\% yield). ${ }^{1} \mathrm{H}$ NMR (400 MHz, $\left.\mathrm{CD}_{3} \mathrm{OD}\right): \delta 1.63\left(\mathrm{~m}, 124,-\mathrm{CH}_{2}-\underline{\mathrm{C}}_{2}-\mathrm{C}_{2}-\mathrm{CH}_{2}-\right), 2.35\left(\mathrm{~m}, 127,-\mathrm{CH}_{2}-\mathrm{CH}_{2}-\mathrm{CH}_{2}-\mathrm{CH}_{2}-\right.$ ), $3.52\left(\mathrm{~m}, 7,-\mathrm{C}_{2}-\mathrm{C} \underline{\mathrm{H}}-\mathrm{C}_{2}{ }^{-}\right), 3.60-3.71$ (broad m, 55, - $\mathrm{C}_{2}-\mathrm{C} \underline{\mathrm{H}}-\mathrm{C} \underline{\mathrm{H}}_{2}-$ ), 3.79 (m, 4, - $\mathrm{C}_{2}-$ $\left.\mathrm{C} \underline{\mathrm{H}}-\mathrm{C} \underline{\mathrm{H}}_{2}-\right), 4.04\left(\mathrm{~m}, 5,-\mathrm{C} \underline{\mathrm{H}}_{2}-\mathrm{C} \underline{\mathrm{H}}-\mathrm{C}_{2}{ }^{-}\right), 4.14\left(\mathrm{~m}, 34,-\underline{\mathrm{C}}_{2}-\mathrm{C} \underline{\mathrm{H}}-\mathrm{C} \underline{\mathrm{H}}_{2}-\right), 4.32\left(\mathrm{~m}, 29,-\mathrm{C}_{2^{-}}\right.$ C $\left.\underline{H}-\underline{C H}_{2}-\right), 5.25$ (m, 14, - $\left.\mathrm{CH}_{2}-\mathrm{C} \underline{H}-\mathrm{CH}_{2}-\right) .{ }^{13} \mathrm{C} \mathrm{NMR}\left(100.6 \mathrm{MHz}, \mathrm{CD}_{3} \mathrm{OD}\right): \delta 173.82$ 
(COOR), 173.63 (COOR), 173.36 (COOR), 173.27 (OOOR), 172.92 (OOOR), 75.45 $(\underline{\mathrm{CH}}), 75.40(\underline{\mathrm{CH}}), 69.96(\underline{\mathrm{CH}}), 69.48(\underline{\mathrm{CH}}), 65.40\left(\mathrm{CH}_{2}\right), 62.92\left(\underline{\mathrm{CH}_{2}}\right), 62.23\left(\mathrm{CH}_{2}\right)$, $60.54\left(\underline{\mathrm{CH}}_{2}\right), 33.53\left(\mathrm{CH}_{2}\right), 33.25\left(\underline{\mathrm{CH}}_{2}\right), 24.15\left(\underline{\mathrm{CH}}_{2}\right)$. FTIR: v $\left(\mathrm{cm}^{-1}\right) 3460(\mathrm{OH}), 2946$ (aliph. C-H stretch), $1730(\mathrm{C}=\mathrm{O})$. MALDI-TOF MS $5975 \mathrm{~m} / \mathrm{z}[\mathrm{M}+\mathrm{Na}]^{+}$(Theory: 5956 m/z [M] $]^{+}$). Elemental Analysis C: $53.43 \%$; H $7.27 \%$ (Theory C: $53.24 \%$; H $7.01 \%$ ). SEC $\mathrm{M}_{\mathrm{w}}: 6880, \mathrm{M}_{\mathrm{n}}: 6780$, PDI: 1.01. $\mathrm{T}_{\mathrm{g}}\left({ }^{\circ} \mathrm{C}\right):-27.6$.

Synthesis of [G4]-PGLAA-bzld (25) - 2-(cis-1,3-O-benzylidene glycerol)adipic acid mono ester (11.81 g, $38.30 \mathrm{mmol})$, [G3]-PGLAA-OH (5.01 g, $0.84 \mathrm{mmol})$, and DPTS (1.62 g, $5.52 \mathrm{mmol})$ were dissolved in THF $(200 \mathrm{~mL})$ followed by the addition of DCC $(11.66 \mathrm{~g}, 56.51 \mathrm{mmol})$. The reaction was stirred at room temperature for 14 hours under nitrogen atmosphere. Upon reaction completion, the DCU was filtered and washed with a small amount of THF $(50 \mathrm{~mL})$. The crude product was purified by silica gel chromatography, eluting with 1.5 to $2.5 \% \mathrm{MeOH}$ in DCM. The appropriate isolated fractions were concentrated, filtered (to remove any DCU), and directly precipitated in hexanes and cooled to $-20{ }^{\circ} \mathrm{C}$ overnight. The hexanes were decanted and the precipitate was isolated to yield $11.21 \mathrm{~g}$ of a sticky wax (87.5\% yield). ${ }^{1} \mathrm{H}$ NMR (400 MHz, $\mathrm{CDCl}_{3}$ ): $\delta 1.63$ (broad m, 260, - $\left.\mathrm{CH}_{2}-\mathrm{C}_{2}-\mathrm{C}_{2}-\mathrm{CH}_{2}-\right), 2.31$ (m, 193, $-\mathrm{C}_{2}-\mathrm{CH}_{2}-\mathrm{CH}_{2}-\underline{\mathrm{C}}_{2}-$ ), $2.41\left(\mathrm{~m}, 67,-\mathrm{C}_{2}-\mathrm{CH}_{2}-\mathrm{CH}_{2}-\mathrm{C}_{2}-\right), 4.10\left(\mathrm{~m}, 132,-\underline{\mathrm{CH}}_{2}-\mathrm{CH}-\mathrm{C}_{2}{ }^{-}\right), 4.24$ (m, 132, - $\underline{\mathrm{CH}}_{2}-$ CH-C $\left.\underline{H}_{2}-\right), 4.67$ (m, 32, - $\left.\mathrm{CH}_{2}-\mathrm{C} \underline{H}-\mathrm{CH}_{2}-\right), 5.19\left(\mathrm{~m}, 30,-\mathrm{CH}_{2}-\mathrm{C} \underline{\mathrm{H}}-\mathrm{CH}_{2}-\right), 5.51$ (s, 32, $\left.\mathrm{C} \underline{\mathrm{H}}\right)$, 7.32 (m, 97, arom. $\mathrm{C} \underline{\mathrm{H}}), 7.46$ (m, 64, arom. $\left.\mathrm{C} \underline{\mathrm{H}}) .{ }^{13} \mathrm{C} \mathrm{NMR} \mathrm{(100.6} \mathrm{MHz,} \mathrm{CDCl}_{3}\right): \delta$ 173.39 (OOOR), 172.87 (OOR), 172.49 (OO), $138.04(\underline{\mathrm{CH}}), 129.27(\underline{\mathrm{CH}}), 128.47$ $(\underline{\mathrm{C}}), 126.22(\underline{\mathrm{CH}}), 101.38(\underline{\mathrm{CH}}), 69.26\left(\underline{\mathrm{C}} \mathrm{H}_{2}\right), 66.11(\underline{\mathrm{CH}}), 62.28\left(\underline{\mathrm{CH}}_{2}\right), 34.08\left(\underline{\mathrm{CH}}_{2}\right)$, $33.83\left(\underline{\mathrm{CH}}_{2}\right), 33.75\left(\underline{\mathrm{CH}}_{2}\right), 33.67\left(\underline{\mathrm{C}} \mathrm{H}_{2}\right), 24.35\left(\underline{\mathrm{CH}}_{2}\right)$. FTIR: $v\left(\mathrm{~cm}^{-1}\right) 2984$ and 2864 
(aliph. C-H stretch), 1731 (C=O). MALDI-TOF MS $15242 \mathrm{~m} / \mathrm{z}$ [M+H] $]^{+}$(Theory: 15246 m/z [M] $]^{+}$). Elemental Analysis C: $60.95 \% ;$ H $6.27 \%$ (Theory C: $61.13 \%$; H $6.55 \%$ ). SEC $\mathrm{M}_{\mathrm{w}}: 11230, \mathrm{M}_{\mathrm{n}}: 11090$, PDI: $1.01 . \mathrm{T}_{\mathrm{g}}\left({ }^{\circ} \mathrm{C}\right): 8.8$.

Synthesis of [G4]-PGLAA-OH (26) - $\mathrm{Pd}(\mathrm{OH})_{2} / \mathrm{C}(20 \% \mathrm{w} / \mathrm{w})$ was added to a solution of [G4]-PGLAA-bzld $(7.70 \mathrm{~g}, 0.505 \mathrm{mmol})$ in THF $(75 \mathrm{~mL})$. The flask for catalytic hydrogenolysis was evacuated and filled with $60 \mathrm{psi}$ of $\mathrm{H}_{2}$ before shaking for 10 hours. The catalyst was filtered and washed with THF solution $(100 \mathrm{~mL})$. The filtrate was evaporated to give $6.02 \mathrm{~g}$ of a clear viscous oil (95.8 \% yield). ${ }^{1} \mathrm{H}$ NMR (400 MHz, $\left.\mathrm{CD}_{3} \mathrm{OD}\right): \delta 1.63\left(\mathrm{~m}, 237,-\mathrm{CH}_{2}-\underline{\mathrm{CH}}_{2}-\underline{\mathrm{CH}}_{2}-\mathrm{CH}_{2}-\right), 2.36\left(\mathrm{~m}, 237,-\mathrm{C}_{2}-\mathrm{CH}_{2}-\mathrm{CH}_{2}-\mathrm{C}_{2}-\right)$, $3.53\left(\mathrm{~m}, 8,-\underline{\mathrm{C}}_{2}-\mathrm{C} \underline{\mathrm{H}}-\underline{\mathrm{C}}_{2}-\right), 3.60-3.71$ (broad m, 112, - $\left.\underline{\mathrm{C}}_{2}-\mathrm{C} \underline{\mathrm{H}}-\mathrm{C} \underline{\mathrm{H}}_{2}-\right), 3.80$ (m, 5, - $\underline{\mathrm{C}}_{2}{ }^{-}$ $\left.\mathrm{C} \underline{\mathrm{H}}-\mathrm{C} \underline{\mathrm{H}}_{2}-\right), 4.04\left(\mathrm{~m}, 5,-\mathrm{C}_{2}-\mathrm{C} \underline{\mathrm{H}}-\mathrm{C}_{2^{-}}\right), 4.17$ (m, 63, - $\left.\underline{\mathrm{CH}}_{2}-\mathrm{C} \underline{\mathrm{H}}-\mathrm{C}_{2}{ }^{-}\right), 4.35$ (m, 58, - $\mathrm{C}_{2^{-}}$ C $\left.\underline{H}-\mathrm{CH}_{2}{ }^{-}\right), 5.25\left(\mathrm{~m}, 30,-\mathrm{CH}_{2}-\mathrm{C} \underline{\mathrm{H}}-\mathrm{CH}_{2}-\right) .{ }^{13} \mathrm{C} \mathrm{NMR}\left(100.6 \mathrm{MHz}, \mathrm{CD}_{3} \mathrm{OD}\right): \delta 173.64$ (COOR), 173.37 (OOR), 173.37 (COOR), 172.93 (OOR), $75.43(\underline{\mathrm{CH}}), 69.46(\underline{\mathrm{CH}})$, $\left.65.42(\underline{\mathrm{CH}})_{2}\right), 62.22\left(\underline{\mathrm{CH}}_{2}\right), 60.55\left(\underline{\mathrm{CH}}_{2}\right), 33.54\left(\underline{\mathrm{CH}}_{2}\right), 33.275\left(\underline{\mathrm{CH}}_{2}\right), 24.15\left(\underline{\mathrm{CH}}_{2}\right)$. FTIR: v $\left(\mathrm{cm}^{-1}\right) 3471(\mathrm{OH}), 2946$ (aliph. C-H stretch), 1729 (C=O). MALDI-TOF MS $12424 \mathrm{~m} / \mathrm{z}$ $[\mathrm{M}+\mathrm{H}]^{+}$(Theory: $12426.57 \mathrm{~m} / \mathrm{z}[\mathrm{M}]^{+}$). Elemental Analysis C: $53.09 \% ; \mathrm{H} 7.11 \%$ (Theory C: $53.35 \%$; H $6.99 \%$ ). SEC $\mathrm{M}_{\mathrm{w}}: 10920, \mathrm{M}_{\mathrm{n}}: 10770$, PDI: 1.01. $\mathrm{T}_{\mathrm{g}}\left({ }^{\circ} \mathrm{C}\right):-30.7$.

Synthesis of [G1]-PGLAA-[G0]-PGLSA-bzld (27) - 2-(cis-1,3-O-benzylidene glycerol)adipic acid mono ester, 6, (11.79 g, 38.25 mmol), [G0]-PGLSA-OH (1.18 g, $4.45 \mathrm{mmol})$, and DPTS (2.85 g, $9.70 \mathrm{mmol})$ were dissolved in THF (50 mL) followed by the addition of DCC (7.22 $\mathrm{g}, 34.97 \mathrm{mmol})$. The reaction was stirred at room temperature for 14 hours under nitrogen atmosphere. Upon completion, the DCU was filtered and washed with a small amount of THF $(50 \mathrm{~mL})$ and the solvent was evaporated. The crude 
product was purified by silica gel chromatography, eluting with 1:1 to 4:1 EtOAc:hexanes. The appropriate isolated fractions were concentrated, filtered (to remove any remaining DCU), and directly precipitated in hexanes and cooled to $-20{ }^{\circ} \mathrm{C}$ overnight. The hexanes were decanted and the precipitate was isolated to yield $7.173 \mathrm{~g}$ of a sticky solid (97\% yield). ${ }^{1} \mathrm{H}$ NMR (400 MHz, $\left.\mathrm{CDCl}_{3}\right): \delta 1.65\left(\mathrm{~m}, 16,-\mathrm{CH}_{2}-\mathrm{C}_{2}-\right.$ $\left.\mathrm{C}_{2}-\mathrm{CH}_{2}-\right), 2.33\left(\mathrm{~m}, 8,-\mathrm{C}_{2}-\mathrm{CH}_{2}-\mathrm{CH}_{2}-\mathrm{C}_{2}-\right), 2.42\left(\mathrm{~m}, 8,-\underline{\mathrm{CH}}_{2}-\mathrm{CH}_{2}-\mathrm{CH}_{2}-\mathrm{C}_{2}-\right), 2.59$ (m, 4, - $\underline{\mathrm{C}}_{2}-\underline{\mathrm{C}}_{2}-$ ), 4.11 (m, 12, - $\underline{\mathrm{C}}_{2}-\mathrm{CH}-\underline{\mathrm{C}}_{2}-$ ), 4.24 (m, 12, - $\underline{\mathrm{C}}_{2}-\mathrm{CH}-\underline{\mathrm{C}}_{2}-$ ), 4.67 (m, 4, $\left.\mathrm{CH}_{2}-\mathrm{C} \underline{H}-\mathrm{CH}_{2}-\right), 5.20$ (m, 2, - $\left.\mathrm{CH}_{2}-\mathrm{C} \underline{\mathrm{H}}-\mathrm{CH}_{2}-\right), 5.51$ (s, 4, $\left.\mathrm{C} \underline{\mathrm{H}}\right), 7.33$ (m, 12, arom. $\left.\mathrm{C} \underline{\mathrm{H}}\right)$, 7.47 (m, 8, arom. $\mathrm{C} \underline{\mathrm{H}}) .{ }^{13} \mathrm{C}$ NMR (100.6 MHz, $\left.\mathrm{CDCl}_{3}\right): \delta 173.41$ (OOR), 172.92 (OOR), 171.48 (ㅁOR), $138.02(\underline{\mathrm{CH}}), 129.28(\underline{\mathrm{CH}}), 128.49(\underline{\mathrm{CH}}), 126.21(\underline{\mathrm{CH}}), 101.38$ $\left.(\underline{\mathrm{C}} \mathrm{H}), 69.65(\underline{\mathrm{CH}}), 69.27\left(\underline{\mathrm{CH}}_{2}\right), 66.11(\underline{\mathrm{CH}}), 62.19(\underline{\mathrm{CH}})_{2}\right), 34.09\left(\underline{\mathrm{C}} \mathrm{H}_{2}\right), 33.73\left(\underline{\mathrm{C}} \mathrm{H}_{2}\right)$, $28.97\left(\underline{\mathrm{CH}}_{2}\right), 24.44\left(\underline{\mathrm{CH}}_{2}\right), 24.36\left(\underline{\mathrm{CH}}_{2}\right)$. FTIR: $v\left(\mathrm{~cm}^{-1}\right) 2948$ and 2862 (aliph. C-H stretch), $1730(\mathrm{C}=\mathrm{O})$. FAB MS $1425.5 \mathrm{~m} / \mathrm{z}[\mathrm{M}+\mathrm{H}]^{+}$(Theory: $\left.1427.49 \mathrm{~m} / \mathrm{z}[\mathrm{M}]^{+}\right)$. Elemental Analysis C: $61.91 \%$; H $6.30 \%$ (Theory C: $62.26 \%$; H $6.35 \%$ ). SEC $\mathrm{M}_{\mathrm{w}}$ : $1670, \mathrm{M}_{\mathrm{n}}: 1650$, PDI: 1.01. $\mathrm{T}_{\mathrm{g}}\left({ }^{\circ} \mathrm{C}\right): 6.3$.

Synthesis of [G1]-PGLAA-[G0]-PGLSA-OH (28) - Pd(OH $)_{2} / \mathrm{C}(20 \%$ w/w) was added to a solution of [G1]-PGLAA-[G0]-PGLSA-bzld (5.90 g, $4.13 \mathrm{mmol})$ in THF (50 mL). The flask for catalytic hydrogenolysis was evacuated and filled with $60 \mathrm{psi}$ of $\mathrm{H}_{2}$ before shaking for 10 hours. The catalyst was filtered and washed with THF $(50 \mathrm{~mL})$. The filtrate was evaporated to give $4.41 \mathrm{~g}$ of a colorless, viscous oil (99\% yield). ${ }^{1} \mathrm{H}$ NMR (400 MHz, $\left.\mathrm{CD}_{3} \mathrm{OD}\right): \delta 1.63\left(\mathrm{~m}, 16,-\mathrm{CH}_{2}-\underline{\mathrm{C}}_{2}-\underline{\mathrm{C}}_{2}-\mathrm{CH}_{2}-\right), 2.36\left(\mathrm{~m}, 16,-\mathrm{CH}_{2}-\mathrm{CH}_{2}-\mathrm{CH}_{2}-\right.$ $\mathrm{C}_{2}-$ ), 2.61 (m, 4, - $\left.\underline{\mathrm{C}}_{2}-\underline{\mathrm{C}}_{2}-\right), 3.52$ (m, 3, - $\underline{\mathrm{C}}_{2}-\mathrm{C} \underline{\mathrm{H}}-\mathrm{C}_{2}{ }^{-}$), 3.59-3.65 (broad m, 9, - $\underline{\mathrm{C}}_{2}{ }^{-}$ $\left.\mathrm{C} \underline{\mathrm{H}}-\mathrm{C} \underline{\mathrm{H}}_{2}-\right), 3.69$ (m, 2, - $\left.\underline{\mathrm{C}}_{2}-\mathrm{C} \underline{\mathrm{H}}-\mathrm{C} \underline{\mathrm{H}}_{2}-\right), 3.79$ (m, 2, - $\left.\underline{\mathrm{C}}_{2}-\mathrm{C} \underline{\mathrm{H}}-\mathrm{C}_{2}-\right), 4.03$ (m, 2, - $\underline{\mathrm{C}}_{2}-$ 
$\left.\mathrm{C} \underline{\mathrm{H}}-\mathrm{C}_{2}{ }^{-}\right), 4.15\left(\mathrm{~m}, 5,-\mathrm{C}_{2}-\mathrm{C} \underline{\mathrm{H}}-\mathrm{C}_{2^{-}}\right), 4.30\left(\mathrm{~m}, 4,-\mathrm{C}_{2}-\mathrm{C} \underline{\mathrm{H}}-\mathrm{C}_{2}-\right), 5.25$ (m, 2, - $\mathrm{CH}_{2^{-}}$ C $\left.\underline{H}-\mathrm{CH}_{2}-\right) .{ }^{13} \mathrm{C}$ NMR (100.6 MHz, $\left.\mathrm{CD}_{3} \mathrm{OD}\right): \delta 173.85$ (COOR), 173.67 (COOR), 173.41

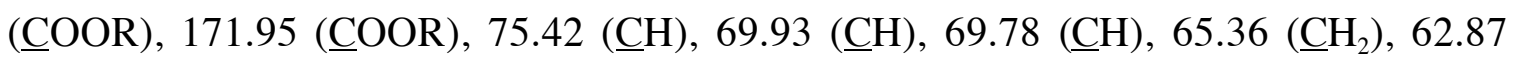
$\left(\mathrm{CH}_{2}\right), 62.04\left(\mathrm{CH}_{2}\right), 60.50\left(\mathrm{CH}_{2}\right), 33.50\left(\mathrm{CH}_{2}\right), 33.29\left(\mathrm{CH}_{2}\right), 33.19\left(\underline{\mathrm{CH}}_{2}\right), 28.61\left(\mathrm{CH}_{2}\right)$, $24.12\left(\mathrm{CH}_{2}\right)$. FTIR: $v\left(\mathrm{~cm}^{-1}\right) 3436(\mathrm{OH}), 2947$ (aliph. C-H stretch), $1727(\mathrm{C}=\mathrm{O})$. MALDITOF MS 1098 m/z [M+Na] $]^{+}$(Theory: 1075 m/z [M] ${ }^{+}$). Elemental Analysis C: $50.96 \%$; H $6.79 \%$ (Theory C: $51.39 \%$; H $6.94 \%$ ). SEC $\mathrm{M}_{\mathrm{w}}: 1680, \mathrm{M}_{\mathrm{n}}: 1660$, PDI: $1.01 . \mathrm{T}_{\mathrm{g}}\left({ }^{\circ} \mathrm{C}\right)$ ) 40.5 .

Synthesis of [G2,G0]-PGLSA-[G1]-PGLAA-bzld (29) - 2-(cis-1,3-O-benzylidene glycerol)succinic acid mono ester, 6, (12.76 g, $45.52 \mathrm{mmol})$, [G1]-PGLAA-[G0]PGLSA-OH (4.28 g, $3.98 \mathrm{mmol})$, and DPTS (5.11 g, $17.38 \mathrm{mmol})$ were dissolved in THF (100 mL) followed by the addition of DCC $(13.91 \mathrm{~g}, 67.44 \mathrm{mmol})$. The reaction was stirred at room temperature for 14 hours under nitrogen atmosphere. Upon completion, the DCU was filtered and washed with a small amount of THF $(50 \mathrm{~mL})$ and the solvent was evaporated. The crude product was purified by silica gel chromatography, eluting with $2 \% \mathrm{MeOH}$ in DCM. The appropriate isolated fractions were concentrated, filtered (to remove any remaining DCU), and directly precipitated in hexanes and cooled to -20 ${ }^{\circ} \mathrm{C}$ overnight. The hexanes were decanted and the precipitate was isolated to yield 10.84 g of a white solid (85.7 \% yield). ${ }^{1} \mathrm{H}$ NMR (400 MHz, $\left.\mathrm{CDCl}_{3}\right): \delta 1.60$ (m, 17, $-\mathrm{CH}_{2}-\mathrm{C}_{2^{-}}$ $\left.\mathrm{CH}_{2}-\mathrm{CH}_{2}-\right), 2.30\left(\mathrm{~m}, 17,-\mathrm{CH}_{2}-\mathrm{CH}_{2}-\mathrm{CH}_{2}-\mathrm{CH}_{2}-\right), 2.63\left(\mathrm{~m}, 20,-\mathrm{C}_{2}-\mathrm{C}_{2}-\right), 2.72(\mathrm{~m}, 16$, -

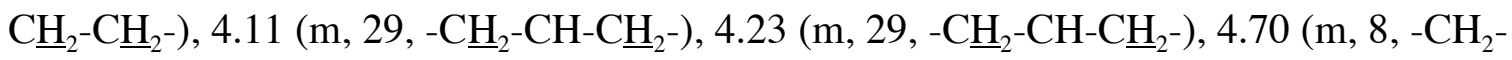
C$\left.-\mathrm{CH}_{2}-\right), 5.20\left(\mathrm{~m}, 6,-\mathrm{CH}_{2}-\mathrm{C} \underline{\mathrm{H}}-\mathrm{CH}_{2}-\right), 5.51$ (s, 8, $\left.\mathrm{C} \underline{\mathrm{H}}\right), 7.34$ (m, 12, arom. $\left.\mathrm{C} \underline{\mathrm{H}}\right), 7.46$ (m,

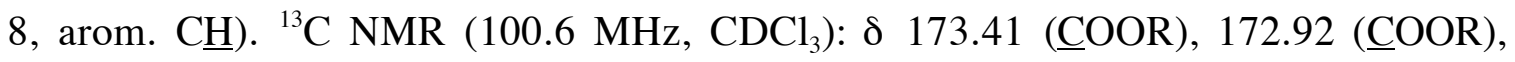


$171.48(\underline{\mathrm{COOR}}), 138.02(\underline{\mathrm{CH}}), 129.28(\underline{\mathrm{CH}}), 128.49(\underline{\mathrm{CH}}), 126.21(\underline{\mathrm{CH}}), 101.38(\underline{\mathrm{CH}})$, $69.65(\underline{\mathrm{CH}}), 69.27\left(\underline{\mathrm{CH}_{2}}\right), 66.11(\underline{\mathrm{C}} \mathrm{H}), 62.19\left(\underline{\mathrm{CH}}_{2}\right), 34.09\left(\underline{\mathrm{CH}}_{2}\right), 33.73\left(\underline{\mathrm{CH}_{2}}\right), 28.97$ $\left(\underline{\mathrm{CH}}_{2}\right), 24.44\left(\mathrm{CH}_{2}\right), 24.36\left(\mathrm{CH}_{2}\right)$. FTIR: $v\left(\mathrm{~cm}^{-1}\right) 2950$ (aliph. C-H stretch), $1732(\mathrm{C}=\mathrm{O})$. FAB MS $3172.7 \mathrm{~m} / \mathrm{z}[\mathrm{M}+\mathrm{H}]^{+}$(Theory: $3173.1 \mathrm{~m} / \mathrm{z}[\mathrm{M}]^{+}$). Elemental Analysis C: 59.61 \%; H $5.84 \%$ (Theory C: $59.81 \%$; H $5.91 \%$ ). SEC $\mathrm{M}_{\mathrm{w}}: 3600, \mathrm{M}_{\mathrm{n}}: 3540$, PDI: $1.02 . \mathrm{T}_{\mathrm{g}}$ $\left({ }^{\circ} \mathrm{C}\right): 32.3$.

Synthesis of [G2,G0]-PGLSA-[G1]-PGLAA-OH (30) - Pd(OH $)_{2} / \mathrm{C}(20 \%$ w/w $)$ was added to a solution of [G2,G0]-PGLSA-[G1]-PGLAA-bzld (5.25 g, $1.65 \mathrm{mmol})$ in THF $(100 \mathrm{~mL})$. The flask for catalytic hydrogenolysis was evacuated and filled with $60 \mathrm{psi}$ of $\mathrm{H}_{2}$ before shaking for 10 hours. The catalyst was filtered and washed with THF $(50 \mathrm{~mL})$. The filtrate was evaporated to give $4.01 \mathrm{~g}$ of a colorless, viscous oil $\left(98.2 \%\right.$ yield). ${ }^{1} \mathrm{H}$ NMR (400 MHz, CD 3 OD): $\delta 1.62\left(\mathrm{~m}, 17,-\mathrm{CH}_{2}-\mathrm{C}_{2}-\mathrm{C}_{2}-\mathrm{CH}_{2}-\right), 2.36$ (m, 17, $-\mathrm{CH}_{2}-\mathrm{CH}_{2}-$ $\left.\mathrm{CH}_{2}-\mathrm{C}_{2}{ }^{-}\right), 2.64\left(\mathrm{~m}, 36,-\mathrm{C}_{2}-\mathrm{C}_{2}{ }^{-}\right), 3.52$ (m, 2, $\left.-\mathrm{C}_{2}-\mathrm{C} \underline{\mathrm{H}}-\mathrm{C}_{2^{-}}{ }^{-}\right), 3.60-3.66$ (broad m, 26, - $\left.\underline{\mathrm{C}}_{2}-\mathrm{C} \underline{\mathrm{H}}-\mathrm{C}_{2}{ }^{-}\right), 3.69$ (m, 9, - $\left.\mathrm{C}_{2}-\mathrm{C} \underline{\mathrm{H}}-\mathrm{C}_{2}{ }^{-}\right), 3.80$ (m, 1, - $\left.\mathrm{C}_{2}-\mathrm{C} \underline{\mathrm{H}}-\mathrm{C}_{2}{ }^{-}\right), 4.18$ (m, 14, - $\left.\mathrm{C}_{2}-\mathrm{C} \underline{\mathrm{H}}-\mathrm{C} \underline{\mathrm{H}}_{2}-\right), 4.32\left(\mathrm{~m}, 12,-\mathrm{C}_{2}-\mathrm{C} \underline{\mathrm{H}}-\mathrm{C}_{2}{ }^{-}\right), 5.25\left(\mathrm{~m}, 6,-\mathrm{CH}_{2}-\mathrm{C} \underline{\mathrm{H}}-\mathrm{CH}_{2}-\right) .{ }^{13} \mathrm{C} \mathrm{NMR}$

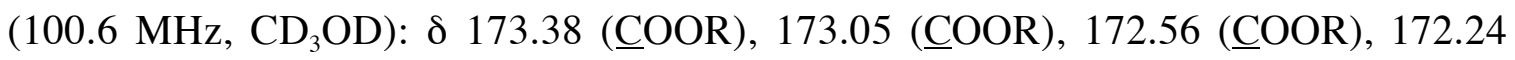
(OOR), 172.00 (OOOR), $75.81(\underline{\mathrm{CH}}), 69.80(\underline{\mathrm{CH}}), 69.35(\underline{\mathrm{CH}}), 67.65\left(\underline{\mathrm{CH}_{2}}\right), 65.68$ $\left(\underline{\mathrm{CH}}_{2}\right), 62.87\left(\underline{\mathrm{CH}}_{2}\right), 62.42\left(\underline{\mathrm{CH}}_{2}\right), 62.11\left(\underline{\mathrm{CH}}_{2}\right), 60.43\left(\underline{\mathrm{CH}}_{2}\right), 33.49\left(\underline{\mathrm{CH}}_{2}\right), 33.20\left(\underline{\mathrm{CH}}_{2}\right)$, $28.83\left(\underline{\mathrm{CH}}_{2}\right), 28.64\left(\underline{\mathrm{CH}}_{2}\right), 25.28\left(\underline{\mathrm{CH}}_{2}\right), 24.09\left(\underline{\mathrm{CH}}_{2}\right)$. FTIR: $v\left(\mathrm{~cm}^{-1}\right) 3468(\mathrm{OH}), 2951$ (aliph. C-H stretch), $1728(\mathrm{C}=\mathrm{O})$. MALDI-TOF MS $2492 \mathrm{~m} / \mathrm{z}$ [M+Na] ${ }^{+}$(Theory: 2468 m/z [M] $]^{+}$). Elemental Analysis C: $50.00 \%$; H $6.40 \%$ (Theory C: $49.63 \%$; H $6.29 \%$ ). SEC $\mathrm{M}_{\mathrm{w}}: 3390, \mathrm{M}_{\mathrm{n}}: 3340$, PDI: $1.02 . \mathrm{T}_{\mathrm{g}}\left({ }^{\circ} \mathrm{C}\right):-22.1$. 
Synthesis of [G3,G1]-PGLAA-[G2,G0]-PGLSA-bzld (31) - 2-(cis-1,3-O-benzylidene glycerol)adipic acid mono ester, 6, (10.75 g, $34.87 \mathrm{mmol})$, [G2,G0]-PGLSA-[G1]PGLAA-OH (3.77 g, $1.53 \mathrm{mmol})$, and DPTS (1.46 g, $4.97 \mathrm{mmol})$ were dissolved in THF $(120 \mathrm{~mL})$ followed by the addition of DCC $(10.60 \mathrm{~g}, 51.38 \mathrm{mmol})$. The reaction was stirred at room temperature for 14 hours under nitrogen atmosphere. Upon completion, the DCC-urea was filtered and washed with a small amount of THF $(50 \mathrm{~mL})$ and the solvent was evaporated. The crude product was purified by silica gel chromatography, eluting with $1.5 \% \mathrm{MeOH}$ in DCM. The appropriate isolated fractions were concentrated, filtered (to remove any remaining DCU), and directly precipitated in hexanes and cooled to $-20{ }^{\circ} \mathrm{C}$ overnight. The hexanes were decanted and the precipitate was isolated to yield $9.88 \mathrm{~g}$ of a sticky solid (90.9 \% yield). ${ }^{1} \mathrm{H} \mathrm{NMR}\left(400 \mathrm{MHz}, \mathrm{CDCl}_{3}\right): \delta 1.65\left(\mathrm{~m}, 81,-\mathrm{CH}_{2}{ }^{-}\right.$ $\left.\mathrm{CH}_{2}-\mathrm{CH}_{2}-\mathrm{CH}_{2}-\right), 2.31$ (m, 52, - $\left.\underline{\mathrm{CH}}_{2}-\mathrm{CH}_{2}-\mathrm{CH}_{2}-\mathrm{C}_{2}{ }^{-}\right), 2.42$ (m, 32, - $\mathrm{C}_{2}-\mathrm{CH}_{2}-\mathrm{CH}_{2}-\mathrm{CH}_{2}-$ ), $2.58\left(\mathrm{~m}, 36-\mathrm{C}_{2}-\underline{\mathrm{CH}}_{2}-\right), 4.10\left(\mathrm{~m}, 62,-\mathrm{C}_{2}-\mathrm{CH}-\underline{\mathrm{H}}_{2}-\right), 4.23$ (m, 62, - $\left.\underline{\mathrm{C}}_{2}-\mathrm{CH}-\mathrm{C}_{2}-\right), 4.66$ (m, 16, - $\left.\mathrm{CH}_{2}-\mathrm{C} \underline{\mathrm{H}}-\mathrm{CH}_{2}-\right), 5.19$ (m, 14, - $\mathrm{CH}_{2}-\mathrm{C} \underline{\mathrm{H}}-\mathrm{CH}_{2}-$ ), 5.51 (s, 16, $\underline{\mathrm{H}}$ ), 7.33 (m, 47, arom. $\mathrm{C} \underline{\mathrm{H}}), 7.46$ (m, 32, arom. $\mathrm{C} \underline{\mathrm{H}}) .{ }^{13} \mathrm{C} \mathrm{NMR}\left(100.6 \mathrm{MHz}, \mathrm{CDCl}_{3}\right): \delta 173.39$ (COOR),

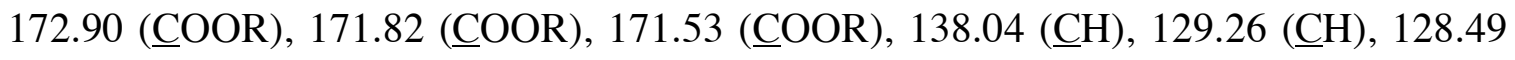
$(\underline{\mathrm{CH}}), 126.22(\underline{\mathrm{CH}}), 101.36(\underline{\mathrm{CH}}), 69.65(\underline{\mathrm{CH}}), 69.26\left(\mathrm{CH}_{2}\right), 66.11(\underline{\mathrm{CH}}), 62.64\left(\mathrm{CH}_{2}\right)$, $\left.62.15(\underline{\mathrm{CH}})_{2}\right), 34.07\left(\underline{\mathrm{CH}}_{2}\right), 33.73\left(\underline{\mathrm{CH}}_{2}\right), 28.96\left(\underline{\mathrm{CH}}_{2}\right), 28.80\left(\underline{\mathrm{CH}}_{2}\right), 24.43\left(\underline{\mathrm{CH}}_{2}\right), 24.35$ $\left(\underline{\mathrm{CH}}_{2}\right)$. FTIR: $v\left(\mathrm{~cm}^{-1}\right) 2948$ and 2862 (aliph. $\mathrm{C}-\mathrm{H}$ stretch), $1731(\mathrm{C}=\mathrm{O})$. MALDI-TOF MS $7137 \mathrm{~m} / \mathrm{z}[\mathrm{M}+\mathrm{Na}]^{+}$(Theory: $7113 \mathrm{~m} / \mathrm{z}[\mathrm{M}]^{+}$). Elemental Analysis C: $60.27 \% ; \mathrm{H} 6.21 \%$ (Theory C: $60.45 \%$; H $6.26 \%$ ). SEC $\mathrm{M}_{\mathrm{w}}: 7160, \mathrm{M}_{\mathrm{n}}: 7060$, PDI: 1.01. $\mathrm{T}_{\mathrm{g}}\left({ }^{\circ} \mathrm{C}\right): 13.4$.

Synthesis of [G3,G1]-PGLAA-[G2,G0]-PGLSA-OH (32) - $\mathrm{Pd}(\mathrm{OH})_{2} / \mathrm{C}(20 \% \mathrm{w} / \mathrm{w})$ was added to a solution of [G3,G1]-PGLAA-[G2,G0]-PGLSA-bzld (9.17 g, $1.29 \mathrm{mmol})$ in 
THF $(100 \mathrm{~mL})$. The flask for catalytic hydrogenolysis was evacuated and filled with 60 psi of $\mathrm{H}_{2}$ before shaking for 10 hours. The catalyst was filtered and washed with THF (50 $\mathrm{mL}$ ). The filtrate was evaporated to give $7.22 \mathrm{~g}$ of a colorless, viscous oil (98.1 \% yield). ${ }^{1} \mathrm{H}$ NMR (400 MHz, CD 3 OD): $\delta 1.63\left(\mathrm{~m}, 83,-\mathrm{CH}_{2}-\mathrm{C}_{2}-\mathrm{C}_{2}-\mathrm{CH}_{2}-\right), 2.37$ (m, 83, - $\underline{\mathrm{C}}_{2}-$ $\left.\mathrm{CH}_{2}-\mathrm{CH}_{2}-\mathrm{C}_{2}{ }^{-}\right), 2.61$ (m, 36, - $\underline{\mathrm{C}}_{2}-\mathrm{C}_{2}-$ ), 3.52 (m, 8, - $\left.\mathrm{C}_{2}-\mathrm{C} \underline{H}-\underline{C H}_{2}-\right)$, 3.60-3.71 (broad $\left.\mathrm{m}, 57,-\underline{\mathrm{C}}_{2}-\mathrm{C} \underline{\mathrm{H}}-\mathrm{C} \underline{\mathrm{H}}_{2}-\right), 3.80$ (m, 4, - $\left.\underline{\mathrm{C}}_{2}-\mathrm{C} \underline{\mathrm{H}}-\underline{\mathrm{C}}_{2}-\right), 4.03$ (m, 5, - $\left.\underline{\mathrm{CH}}_{2}-\mathrm{C} \underline{\mathrm{H}}-\mathrm{C} \underline{\mathrm{H}}_{2}-\right), 4.11-$ $4.23\left(\mathrm{~m}, 34,-\underline{\mathrm{C}}_{2}-\mathrm{C} \underline{\mathrm{H}}-\mathrm{C}_{2}{ }^{-}\right), 4.30$ (m, 29, - $\left.\underline{\mathrm{CH}}_{2}-\mathrm{C} \underline{\mathrm{H}}-\underline{\mathrm{C}}_{2}{ }^{-}\right), 5.25$ (m, 14, - $\left.\mathrm{CH}_{2}-\mathrm{C} \underline{\mathrm{H}}-\mathrm{CH}_{2}-\right)$. ${ }^{13} \mathrm{C}$ NMR (100.6 MHz, $\left.\mathrm{CD}_{3} \mathrm{OD}\right): \delta 173.85$ (COOR), 173.67 (COOR), 173.41 (COOR), 171.95 (ㅁOR), $75.42(\underline{\mathrm{CH}}), 69.93(\underline{\mathrm{C}} \mathrm{H}), 69.78(\underline{\mathrm{CH}}), 65.36\left(\underline{\mathrm{CH}_{2}}\right), 62.87\left(\underline{\mathrm{C}} \mathrm{H}_{2}\right), 62.04$ $\left(\underline{\mathrm{CH}}_{2}\right), 60.50\left(\underline{\mathrm{CH}}_{2}\right), 33.50\left(\underline{\mathrm{CH}}_{2}\right), 33.29\left(\underline{\mathrm{CH}}_{2}\right), 33.19\left(\underline{\mathrm{CH}}_{2}\right), 28.61\left(\underline{\mathrm{CH}}_{2}\right), 24.12\left(\underline{\mathrm{CH}}_{2}\right)$. FTIR: $v\left(\mathrm{~cm}^{-1}\right) 3463(\mathrm{OH}), 2947$ (aliph. C-H stretch), $1729(\mathrm{C}=\mathrm{O})$. MALDI-TOF MS $5730 \mathrm{~m} / \mathrm{z}[\mathrm{M}+\mathrm{Na}]^{+}$(Theory: $5703 \mathrm{~m} / \mathrm{z}[\mathrm{M}]^{+}$). Elemental Analysis C: $51.91 \% ; \mathrm{H} 6.93 \%$ (Theory C: $51.80 \%$; H $6.68 \%$ ). SEC $\mathrm{M}_{\mathrm{w}}: 6570, \mathrm{M}_{\mathrm{n}}: 6490$, PDI: $1.01 . \mathrm{T}_{\mathrm{g}}\left({ }^{\circ} \mathrm{C}\right):-23.8$. Synthesis of $[\mathbf{G 4 , G 2 , G 0 ] - P G L S A - [ G 3 , G 1 ] - P G L A A - b z l d ~ ( 3 3 ) ~ - ~ 2 - ( c i s - 1 , 3 - O - ~}$ benzylidene glycerol)succinic acid mono ester, 6, (11.57 g, $41.28 \mathrm{mmol})$, [G3,G1]PGLAA-[G2,G0]-PGLSA-OH (5.59 g, $0.98 \mathrm{mmol})$, and DPTS (4.09 g, $13.92 \mathrm{mmol})$ were dissolved in THF ( $80 \mathrm{~mL}$ ) followed by the addition of DCC (12.60 g, $61.05 \mathrm{mmol})$. The reaction was stirred at room temperature for 14 hours under nitrogen atmosphere. Upon completion, the DCC-urea was filtered and washed with a small amount of THF $(50 \mathrm{~mL})$ and the solvent was evaporated. The crude product was purified by silica gel chromatography, eluting with $1.5 \%$ to $5.0 \% \mathrm{MeOH}$ in DCM. The appropriate isolated fractions were concentrated, filtered (to remove any remaining DCU), and directly precipitated in hexanes and cooled to $-20{ }^{\circ} \mathrm{C}$ over 48 hours. The hexanes were decanted 
and the precipitate was isolated to yield $11.50 \mathrm{~g}$ of a white solid (83.2\% yield). ${ }^{1} \mathrm{H}$ NMR (400 MHz, $\mathrm{CDCl}_{3}$ ): $\delta 1.59\left(\mathrm{~m}, 83,-\mathrm{CH}_{2}-\mathrm{CH}_{2}-\mathrm{C}_{2}-\mathrm{CH}_{2}-\right), 2.30$ (m, 83, - $\mathrm{CH}_{2}-\mathrm{CH}_{2}-\mathrm{CH}_{2}-$

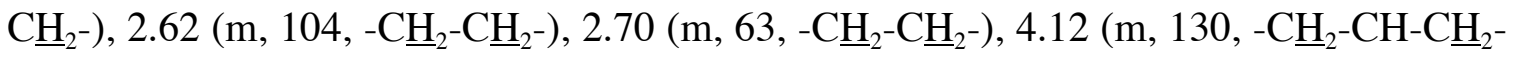
), $4.22\left(\mathrm{~m}, 130,-\mathrm{C}_{2}-\mathrm{CH}-\mathrm{C}_{2}{ }^{-}\right), 4.68\left(\mathrm{~m}, 32,-\mathrm{CH}_{2}-\mathrm{C} \underline{\mathrm{H}}-\mathrm{CH}_{2}-\right), 5.18$ (m, 30, - $\mathrm{CH}_{2}-\mathrm{C} \underline{\mathrm{H}}-$ $\left.\mathrm{CH}_{2^{-}}\right), 5.50$ (s, 32, $\left.\mathrm{C} \underline{\mathrm{H}}\right), 7.33$ (m, 97, arom. $\left.\mathrm{C} \underline{\mathrm{H}}\right), 7.46$ (m, 66, arom. $\left.\underline{\mathrm{C}}\right) .{ }^{13} \mathrm{C}$ NMR

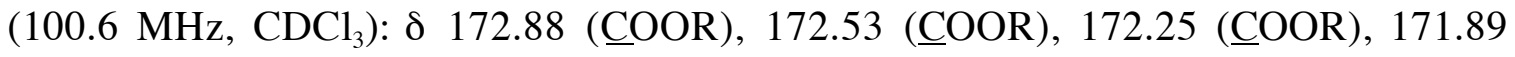
(ㅁOR), $138.04(\underline{\mathrm{CH}}), 129.26(\underline{\mathrm{CH}}), 128.48(\underline{\mathrm{CH}}), 126.22(\underline{\mathrm{CH}}), 101.28(\underline{\mathrm{CH}}), 69.14$ $\left(\underline{\mathrm{C}} \mathrm{H}_{2}\right), 66.54(\underline{\mathrm{C}} \mathrm{H}), 62.60\left(\underline{\mathrm{C}} \mathrm{H}_{2}\right), 33.81\left(\underline{\mathrm{C}} \mathrm{H}_{2}\right), 33.66\left(\underline{\mathrm{CH}}_{2}\right), 29.35\left(\underline{\mathrm{C}} \mathrm{H}_{2}\right), 29.03\left(\underline{\mathrm{C}} \mathrm{H}_{2}\right)$, $24.30\left(\underline{\mathrm{CH}}_{2}\right)$. FTIR: $v\left(\mathrm{~cm}^{-1}\right) 2959$ (aliph. C-H stretch), $1732(\mathrm{C}=\mathrm{O})$. MALDI-TOF MS $14082 \mathrm{~m} / \mathrm{z}[\mathrm{M}+\mathrm{H}]^{+}$(Theory: 14096 m/z [M] $]^{+}$. Elemental Analysis C: $59.17 \%$; H $6.07 \%$ (Theory C: $59.13 \%$; H $5.916 \%$ ). SEC $\mathrm{M}_{\mathrm{w}}: 10440, \mathrm{M}_{\mathrm{n}}: 10290$, PDI: $1.02 . \mathrm{T}_{\mathrm{g}}\left({ }^{\circ} \mathrm{C}\right): 32.7$ at half-height.

Synthesis of $[\text { G4,G2,G0]-PGLSA-[G3,G1]-PGLAA-OH (34) - Pd(OH })_{2} / \mathrm{C}(20 \%$ w/w) was added to a solution of [G4,G2,G0]-PGLSA-[G3,G1]-PGLAA-bzld (2.08 g, 0.15 mmol) in THF $(80 \mathrm{~mL})$. The flask for catalytic hydrogenolysis was evacuated and filled with 60 psi of $\mathrm{H}_{2}$ before shaking for 10 hours. The catalyst was filtered and washed with THF (75 mL). The filtrate was evaporated to give $1.65 \mathrm{~g}$ of a colorless, viscous oil (99.1 $\%$ yield). ${ }^{1} \mathrm{H}$ NMR (400 MHz, $\left.\mathrm{CD}_{3} \mathrm{OD}\right): \delta 1.62\left(\mathrm{~m}, 80,-\mathrm{CH}_{2}-\mathrm{C}_{2}-\mathrm{C}_{2}-\mathrm{CH}_{2}-\right), 2.37(\mathrm{~m}$, 80, - $\left.\underline{\mathrm{C}}_{2}-\mathrm{CH}_{2}-\mathrm{CH}_{2}-\mathrm{C}_{2}{ }^{-}\right), 2.64$ (m, 164, - $\left.\mathrm{C}_{2}-\mathrm{C}_{2}-\right), 3.52$ (m, 12, - $\left.\underline{\mathrm{CH}}_{2}-\mathrm{C} \underline{\mathrm{H}}-\mathrm{C}_{2}{ }^{-}\right), 3.63-$ 3.71 (broad m, 160, - $\underline{\mathrm{C}}_{2}-\mathrm{C} \underline{\mathrm{H}}-\mathrm{C}_{2^{-}}$), 3.80 (m, 6, - $\left.\underline{\mathrm{C}}_{2}-\mathrm{C} \underline{\mathrm{H}}-\underline{\mathrm{C}}_{2}-\right), 4.06$ (m, 14, - $\mathrm{C} \underline{\mathrm{H}}_{2}-\mathrm{C} \underline{\mathrm{H}}-$ $\left.\mathrm{C}_{2^{-}}\right), 4.20\left(\mathrm{~m}, 62,-\mathrm{C}_{2}-\mathrm{C} \underline{\mathrm{H}}-\mathrm{C} \underline{\mathrm{H}}_{2}{ }^{-}\right), 4.30$ (m, 60, - $\left.\underline{\mathrm{CH}}_{2}-\mathrm{C} \underline{\mathrm{H}}-\mathrm{C}_{2^{-}}\right), 5.25$ (m, 30, - $\mathrm{CH}_{2^{-}}$

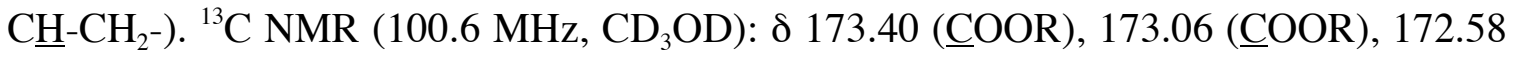
(ㅁOR), $75.82(\underline{\mathrm{C}} \mathrm{H}), 69.90(\underline{\mathrm{CH}}), 69.34(\underline{\mathrm{CH}}), 67.64\left(\underline{\mathrm{CH}}_{2}\right), 62.45\left(\underline{\mathrm{CH}_{2}}\right), 62.15\left(\underline{\mathrm{C}} \mathrm{H}_{2}\right)$, 
$60.46\left(\underline{\mathrm{C}} \mathrm{H}_{2}\right), 33.25\left(\underline{\mathrm{CH}}_{2}\right), 28.87\left(\underline{\mathrm{CH}}_{2}\right), 28.67\left(\underline{\mathrm{CH}}_{2}\right), 25.27\left(\underline{\mathrm{CH}}_{2}\right), 24.12\left(\underline{\mathrm{CH}}_{2}\right)$. FTIR: v $\left(\mathrm{cm}^{-1}\right) 3441(\mathrm{OH}), 2948$ (aliph. C-H stretch), 1728 (C=O). MALDI-TOF MS 11299 m/z $[\mathrm{M}+\mathrm{Na}]^{+}$(Theory: $11276 \mathrm{~m} / \mathrm{z}[\mathrm{M}]^{+}$). Elemental Analysis C: $50.49 \%$; H $6.41 \%$ (Theory C: $50.06 \%$; H $6.24 \%)$. SEC $\mathrm{M}_{\mathrm{w}}: 9150, \mathrm{M}_{\mathrm{n}}: 9000$, PDI: 1.02. $\mathrm{T}_{\mathrm{g}}\left({ }^{\circ} \mathrm{C}\right):-21.6$.

Synthesis of [G3]-PGLSA-[G2,G1,G0]-PGLAA-bzld (35) - [G2]-PGLAA-OH $(0.52$ $\mathrm{g}, 0.19 \mathrm{mmol})$ was dissolved in THF $(30 \mathrm{~mL})$ followed by the addition of 2-(cis-1,3-Obenzylidene glycerol)succinic acid mono ester anhydride (2.27 g, $4.18 \mathrm{mmol})$ and DMAP $(0.07 \mathrm{~g}, 0.57 \mathrm{mmol})$ respectively. The reaction was stirred at room temperature for 14 hours under nitrogen atmosphere. Any remaining anhydride was quenched by the addition of water $(1.0 \mathrm{~mL})$ that was allowed to stir for another 5 hours. The THF and water were removed under vacuum. The crude product was purified by silica gel chromatography, eluting with $1.0 \%$ to $3.0 \% \mathrm{MeOH}$ in DCM. The isolated fractions contained 2-(cis-1,3-O-benzylidene glycerol)succinic acid mono ester so the remaining contents were dissolved in DCM $(125 \mathrm{~mL})$ and washed once with $0.1 \mathrm{~N} \mathrm{HCl}(125 \mathrm{~mL})$ and three times with saturated sodium bicarbonate $(125 \mathrm{~mL})$. The organic phase was dried with $\mathrm{Na}_{2} \mathrm{SO}_{4}$, filtered, and concentrated to yield $1.15 \mathrm{~g}$ of a white solid $(87.3 \%$ yield). ${ }^{1} \mathrm{H} \mathrm{NMR}\left(400 \mathrm{MHz}, \mathrm{CDCl}_{3}\right): \delta 1.60\left(\mathrm{~m}, 52,-\mathrm{CH}_{2}-\mathrm{C}_{2}-\mathrm{C}_{2}-\mathrm{CH}_{2}-\right), 2.30$ (m, 52, $\left.\mathrm{CH}_{2}-\mathrm{CH}_{2}-\mathrm{CH}_{2}-\mathrm{CH}_{2}-\right), 2.63\left(\mathrm{~m}, 33-\mathrm{C}_{2}-\mathrm{C}_{2}-\right), 2.70$ (m, $\left.33-\mathrm{CH}_{2}-\mathrm{CH}_{2}-\right), 4.12$ (m, 66, $\left.\mathrm{C}_{2}-\mathrm{CH}-\mathrm{C} \underline{\mathrm{H}}_{2}-\right), 4.24\left(\mathrm{~m}, 66,-\underline{\mathrm{CH}}_{2}-\mathrm{CH}-\mathrm{C}_{2}-\right), 4.69\left(\mathrm{~m}, 16,-\mathrm{CH}_{2}-\mathrm{C} \underline{\mathrm{H}}-\mathrm{CH}_{2}-\right), 5.20(\mathrm{~m}, 13$, $\left.-\mathrm{CH}_{2}-\mathrm{C} \underline{\mathrm{H}}-\mathrm{CH}_{2}-\right), 5.50$ (s, 16, $\left.\mathrm{C} \underline{\mathrm{H}}\right), 7.33$ (m, 54, arom. $\left.\mathrm{C} \underline{\mathrm{H}}\right), 7.46$ (m, 35, arom. $\left.\mathrm{C} \underline{\mathrm{H}}\right) .{ }^{13} \mathrm{C}$

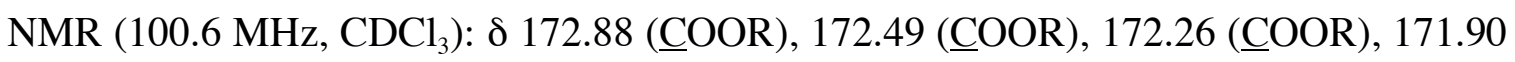
(ㅁOR), $138.01(\underline{\mathrm{C}} \mathrm{H}), 129.26(\underline{\mathrm{CH}}), 128.48(\underline{\mathrm{CH}}), 126.21(\underline{\mathrm{C}} \mathrm{H}), 101.30(\underline{\mathrm{CH}}), 69.15$ $(\underline{\mathrm{C}}), 66.54(\underline{\mathrm{CH}}), 62.62\left(\underline{\mathrm{C}} \mathrm{H}_{2}\right), 62.28\left(\underline{\mathrm{C}} \mathrm{H}_{2}\right), 33.82\left(\underline{\mathrm{CH}}_{2}\right), 33.67\left(\underline{\mathrm{CH}}_{2}\right), 29.36\left(\underline{\mathrm{CH}}_{2}\right)$, 
$29.03\left(\underline{\mathrm{CH}}_{2}\right), 24.30\left(\underline{\mathrm{CH}}_{2}\right)$. FTIR: $v\left(\mathrm{~cm}^{-1}\right) 2961$ (aliph. C-H stretch), $1732(\mathrm{C}=\mathrm{O})$. MALDI-TOF MS $6920 \mathrm{~m} / \mathrm{z}[\mathrm{M}+\mathrm{H}]^{+}$(Theory: $6917 \mathrm{~m} / \mathrm{z}[\mathrm{M}]^{+}$). Elemental Analysis C: $59.58 \%$; H $5.95 \%$ (Theory C: $59.73 \%$; H 6.03\%). SEC $\mathrm{M}_{\mathrm{w}}: 6580, \mathrm{M}_{\mathrm{n}}: 6500$, PDI: 1.01. $\mathrm{T}_{\mathrm{g}}\left({ }^{\circ} \mathrm{C}\right): 28.0$.

Synthesis of [G3]-PGLSA-[G2,G1,G0]-PGLAA-OH (36) - $\mathrm{Pd}(\mathrm{OH})_{2} / \mathrm{C}(20 \%$ w/w) was added to a solution of [G3]-PGLSA-[G2,G1,G0]-PGLAA-bzld (0.96 g, $0.14 \mathrm{mmol})$ in THF $(50 \mathrm{~mL})$. The flask for catalytic hydrogenolysis was evacuated and filled with $60 \mathrm{psi}$ of $\mathrm{H}_{2}$ before shaking for 10 hours. The catalyst was filtered and washed with THF (50 $\mathrm{mL}$ ). The filtrate was evaporated to give $0.73 \mathrm{~g}$ of a colorless, viscous oil ( $95.4 \%$ yield). ${ }^{1} \mathrm{H}$ NMR (400 MHz, CD 3 OD): $\delta 1.62\left(\mathrm{~m}, 52,-\mathrm{CH}_{2}-\underline{\mathrm{CH}}_{2}-\underline{\mathrm{C}}_{2}-\mathrm{CH}_{2}-\right.$ ), 2.35 (m, 52, - $\underline{\mathrm{C}}_{2}-$

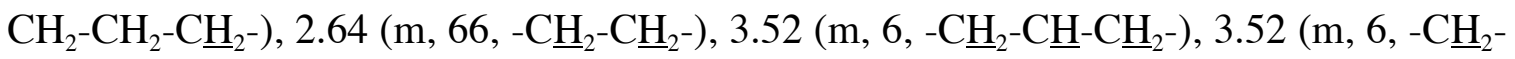
$\left.\mathrm{C} \underline{\mathrm{H}}-\mathrm{C} \underline{\mathrm{H}}_{2}-\right), 3.65\left(\mathrm{~m}, 58,-\mathrm{C}_{2}-\mathrm{C} \underline{\mathrm{H}}-\underline{\mathrm{C}}_{2}-\right), 3.80\left(\mathrm{~m}, 4,-\mathrm{C}_{2}-\mathrm{C} \underline{\mathrm{H}}-\mathrm{C} \underline{\mathrm{H}}_{2}-\right), 4.05\left(\mathrm{~m}, 4,-\underline{\mathrm{C}}_{2}-\right.$

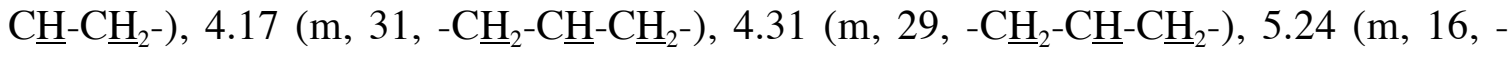
$\left.\mathrm{CH}_{2}-\mathrm{C} \underline{H}-\mathrm{CH}_{2}-\right) .{ }^{13} \mathrm{C}$ NMR (100.6 MHz, $\left.\mathrm{CD}_{3} \mathrm{OD}\right): \delta 173.36$ (COOR), 172.98 (OOR), 172.58 (ㅁOR), $75.81(\underline{\mathrm{CH}}), 69.48(\underline{\mathrm{CH}}), 62.43\left(\underline{\mathrm{C}} \mathrm{H}_{2}\right), 60.42\left(\underline{\mathrm{CH}}_{2}\right), 33.23\left(\underline{\mathrm{CH}_{2}}\right), 28.82$ $\left(\underline{\mathrm{CH}}_{2}\right), 28.64\left(\underline{\mathrm{CH}}_{2}\right), 24.09\left(\underline{\mathrm{CH}}_{2}\right)$. FTIR: $v\left(\mathrm{~cm}^{-1}\right) 3467(\mathrm{OH}), 2946$ (aliph. C-H stretch), $1729(\mathrm{C}=\mathrm{O})$. MALDI-TOF MS $5529 \mathrm{~m} / \mathrm{z}[\mathrm{M}+\mathrm{Na}]^{+}$(Theory: $\left.5507 \mathrm{~m} / \mathrm{z}[\mathrm{M}]^{+}\right)$. Elemental Analysis C: $50.22 \%$; H $6.58 \%$ (Theory C: $50.60 \%$; H $6.41 \%$ ). SEC $\mathrm{M}_{\mathrm{w}}:$ 5900, $\mathrm{M}_{\mathrm{n}}$ : 5820, PDI: $1.01 . \mathrm{T}_{\mathrm{g}}\left({ }^{\circ} \mathrm{C}\right):-24.1$.

Synthesis of [G4,G3]-PGLSA-[G2,G1,G0]-PGLAA-bzld (37) - [G3]-PGLSA[G2,G1,G0]-PGLAA-OH (0.51 g, $0.09 \mathrm{mmol})$ was dissolved in THF/dimethyl acetamide (9:1) $(25 \mathrm{~mL})$ followed by the addition of 2-(cis-1,3-O-benzylidene glycerol)succinic acid mono ester anhydride, 4, (2.72 g, $5.01 \mathrm{mmol})$ and DMAP (0.06 g, $0.46 \mathrm{mmol})$ 
respectively. The reaction was stirred at room temperature for 14 hours under nitrogen atmosphere. Any remaining anhydride was quenched by the addition of water $(1.0 \mathrm{~mL})$ that was allowed to stir for another 5 hours. The THF and water were removed under vacuum and the remaining contents were dissolved in DCM $(125 \mathrm{~mL})$ and washed once with $0.1 \mathrm{~N} \mathrm{HCl}(125 \mathrm{~mL})$ and three times with saturated sodium bicarbonate $(125 \mathrm{~mL})$. The organic phase was dried with $\mathrm{Na}_{2} \mathrm{SO}_{4}$, filtered, and concentrated before the dendrimer was precipitated in hexanes $(500 \mathrm{~mL})$ and cooled to $-20{ }^{\circ} \mathrm{C}$ overnight. The hexanes were decanted and the precipitate was isolated to yield $1.151 \mathrm{~g}$ of a white solid (90.0\% yield). ${ }^{1} \mathrm{H} \mathrm{NMR}\left(400 \mathrm{MHz}, \mathrm{CDCl}_{3}\right): \delta 1.60$ (m, 52, $\left.-\mathrm{CH}_{2}-\mathrm{C}_{2}-\mathrm{C}_{2}-\mathrm{CH}_{2}-\right), 2.30$ (m, 52, $-\mathrm{C}_{2}-\mathrm{CH}_{2}-\mathrm{CH}_{2}-\mathrm{C}_{2}-$ ), 2.58-2.64 (broad m, $70-\underline{\mathrm{CH}}_{2}-\underline{\mathrm{CH}}_{2}-$ ), 2.70 (m, $130-\mathrm{C}_{2}-$ $\left.\mathrm{C}_{2^{-}}-\right), 4.11\left(\mathrm{~m}, 126,-\underline{\mathrm{CH}}_{2}-\mathrm{CH}-\underline{\mathrm{CH}}_{2}{ }^{-}\right), 4.22\left(\mathrm{~m}, 126,-\underline{\mathrm{CH}}_{2}-\mathrm{CH}-\mathrm{C} \underline{\mathrm{H}}_{2}{ }^{-}\right), 4.68\left(\mathrm{~m}, 32,-\mathrm{CH}_{2}-\right.$ C$\left.-\mathrm{CH}_{2}-\right), 5.19$ (m, 30, - $\left.\mathrm{CH}_{2}-\mathrm{C} \underline{H}-\mathrm{CH}_{2}-\right), 5.49$ (s, 32, $\left.\mathrm{C} \underline{\mathrm{H}}\right), 7.32$ (m, 98, arom. $\left.\mathrm{C} \underline{\mathrm{H}}\right), 7.45$

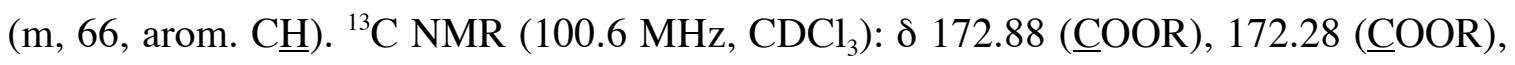

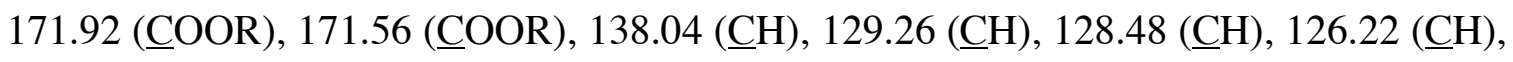
$101.29(\underline{\mathrm{CH}}), 69.51(\underline{\mathrm{CH}}), 69.14(\underline{\mathrm{CH}}), 66.54(\underline{\mathrm{CH}}), 62.46\left(\underline{\mathrm{CH}}_{2}\right), 33.65\left(\underline{\mathrm{CH}_{2}}\right), 29.35$ $\left(\underline{\mathrm{CH}}_{2}\right), 29.02\left(\mathrm{CH}_{2}\right), 24.29\left(\underline{\mathrm{CH}}_{2}\right)$. FTIR: $v\left(\mathrm{~cm}^{-1}\right) 2955$ and 2859 (aliph. C-H stretch), $1732(\mathrm{C}=\mathrm{O})$. MALDI-TOF MS $13903 \mathrm{~m} / \mathrm{z}[\mathrm{M}+\mathrm{H}]^{+}$(Theory: $13899 \mathrm{~m} / \mathrm{z}[\mathrm{M}]^{+}$). Elemental Analysis C: $58.48 \%$; H $5.83 \%$ (Theory C: $58.76 \%$; H $5.79 \%$ ). SEC $\mathrm{M}_{\mathrm{w}}:$ 10480, $\mathrm{M}_{\mathrm{n}}$ : 10310, PDI: $1.02 . \mathrm{T}_{\mathrm{g}}\left({ }^{\circ} \mathrm{C}\right): 36.2$.

Synthesis of [G4,G3]-PGLSA-[G2,G1,G0]-PGLAA-OH (38) - Pd(OH $)_{2} / \mathrm{C}(20 \%$ w/w) was added to a solution of [G4,G3]-PGLSA-[G2,G1,G0]-PGLAA-bzld (0.90 g, 0.06 mmol) in THF $(50 \mathrm{~mL})$. The flask for catalytic hydrogenolysis was evacuated and filled with 60 psi of $\mathrm{H}_{2}$ before shaking for 10 hours. The catalyst was filtered and washed with 
THF (75 mL). The filtrate was evaporated to give $0.68 \mathrm{~g}$ of a colorless, viscous oil (94.6 $\%$ yield). ${ }^{1} \mathrm{H}$ NMR (400 MHz, $\left.\mathrm{CD}_{3} \mathrm{OD}\right): \delta 1.63$ (m, 52, - $\left.\mathrm{CH}_{2}-\mathrm{CH}_{2}-\mathrm{C}_{2}-\mathrm{CH}_{2}-\right), 2.37$ (m, 52, - $\left.\underline{\mathrm{C}}_{2}-\mathrm{CH}_{2}-\mathrm{CH}_{2}-\mathrm{C}_{2}{ }^{-}\right), 2.65$ (m, 194, - $\left.\mathrm{C}_{2}-\mathrm{C}_{2}-\right), 3.53$ (m, 14, - $\left.\underline{\mathrm{C}}_{2}-\mathrm{C} \underline{\mathrm{H}}-\underline{\mathrm{C}}_{2}-\right), 3.65$ (m, 108, - $\left.\underline{\mathrm{CH}}_{2}-\mathrm{C} \underline{\mathrm{H}}-\mathrm{C} \underline{\mathrm{H}}_{2}-\right), 3.80$ (m, 5, - $\left.\underline{\mathrm{CH}}_{2}-\mathrm{C} \underline{\mathrm{H}}-\underline{\mathrm{CH}}_{2}-\right), 4.05$ (m, 8, - $\left.\underline{\mathrm{CH}}_{2}-\mathrm{C} \underline{\mathrm{H}}-\underline{\mathrm{CH}}_{2}-\right), 4.15$ (m, 14, - $\left.\underline{\mathrm{C}}_{2}-\mathrm{C} \underline{\mathrm{H}}-\underline{\mathrm{CH}}_{2}-\right), 4.23$ (m, 54, - $\left.\underline{\mathrm{CH}}_{2}-\mathrm{C} \underline{\mathrm{H}}-\underline{\mathrm{C}}_{2}-\right), 4.31$ (m, 60, - $\left.\underline{\mathrm{C}}_{2}-\mathrm{C} \underline{\mathrm{H}}-\mathrm{C}_{2}{ }^{-}\right), 5.26$ (m, 30, $\left.-\mathrm{CH}_{2}-\mathrm{C} \underline{\mathrm{H}}-\mathrm{CH}_{2}-\right) .{ }^{13} \mathrm{C}$ NMR (100.6 MHz, $\mathrm{CD}_{3} \mathrm{OD}$ ): $\delta 173.43$ (OOR), 172.67

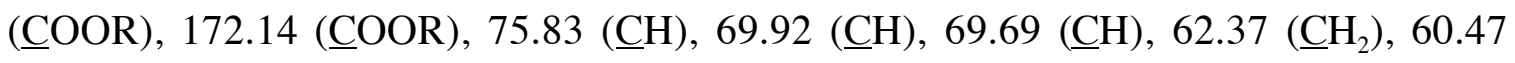
$\left(\underline{\mathrm{CH}}_{2}\right), 33.29\left(\underline{\mathrm{CH}}_{2}\right), 28.89\left(\underline{\mathrm{CH}}_{2}\right), 28.69\left(\underline{\mathrm{CH}}_{2}\right), 24.13\left(\underline{\mathrm{CH}}_{2}\right)$. FTIR: v $\left(\mathrm{cm}^{-1}\right) 3452(\mathrm{OH})$, 2950 (aliph. C-H stretch), 1729 (C=O). MALDI-TOF MS $11084 \mathrm{~m} / \mathrm{z}[\mathrm{M}+\mathrm{H}]^{+}$(Theory: $11080 \mathrm{~m} / \mathrm{z}[\mathrm{M}]^{+}$). Elemental Analysis C: $49.25 \%$; H $6.38 \%$ (Theory C: $49.43 \%$; H 6.09 \%). SEC $\mathrm{M}_{\mathrm{w}}: 8310, \mathrm{M}_{\mathrm{n}}: 8160$, PDI: $1.02 . \mathrm{T}_{\mathrm{g}}\left({ }^{\circ} \mathrm{C}\right):-21.2$.

QELS. A variety of solvent concentrations were used to obtain satisfactory QELS correlation functions. In general, $50 \mathrm{mg}$ of dendrimer in $0.5 \mathrm{~mL}$ of solvent produced the best correlation functions. At least fifteen measurements were recorded for each dendrimer with good correlations and up to thirty measurements were recorded for dendrimers with more variation. The values reported are the average of the recorded values with standard deviation. 